Pacific

Journal of

Mathematics

NONSPLITTABILITY OF THE RATIONAL HOMOLOGY COBORDISM GROUP OF 3-MANIFOLDS

SE-Goo Kim AND CHARles Livingston

Volume $271 \quad$ No. 1

September 2014 


\title{
NONSPLITTABILITY OF THE RATIONAL HOMOLOGY COBORDISM GROUP OF 3-MANIFOLDS
}

\author{
Se-Goo Kim AND ChaRles LivingSton
}

\begin{abstract}
Let $\mathbb{Z}[1 / p]$ denote the ring of integers with the prime $p$ inverted. There is a canonical homomorphism $\Psi: \bigoplus \Theta_{\mathbb{Z}[1 / p]}^{3} \rightarrow \Theta_{\mathbb{Q}}^{3}$, where $\Theta_{R}^{3}$ denotes the three-dimensional smooth $R$-homology cobordism group of $R$-homology spheres and the direct sum is over all prime integers. Gauge-theoretic methods prove the kernel is infinitely generated. Here we prove that $\Psi$ is not surjective, with cokernel infinitely generated. As a basic example we show that for $p$ and $q$ distinct primes, there is no rational homology cobordism from the lens space $L(p q, 1)$ to any $M_{p} \# M_{q}$, where $H_{1}\left(M_{p}\right)=\mathbb{Z}_{p}$ and $H_{1}\left(M_{q}\right)=\mathbb{Z}_{q}$. More subtle examples include cases in which a cobordism to such a connected sum exists topologically but not smoothly. (Conjecturally such a splitting always exists topologically.) Further examples can be chosen to represent 2-torsion in $\Theta_{\mathbb{Q}}^{3}$. Let $\mathscr{K}$ denote the kernel of $\Theta_{\mathbb{Q}}^{3} \rightarrow \widehat{\Theta}_{\mathbb{Q}}^{3}$, where $\widehat{\boldsymbol{\Theta}}_{\mathbb{Q}}^{3}$ denotes the topological homology cobordism group. Freedman proved that $\Theta_{\mathbb{Z}}^{\mathbf{3}} \subset \mathscr{K}$. A corollary of results here is that $\mathscr{K} / \Theta_{\mathbb{Z}}^{\mathbf{3}}$ is infinitely generated. We also demonstrate the failure in dimension three of splitting theorems that apply to higher-dimensional knot concordance groups.
\end{abstract}

\section{Introduction}

Furuta [1990] applied instanton gauge theory to reveal unexpectedly deep structure in the homology cobordism group of smooth homology 3 -spheres, $\Theta_{\mathbb{Z}}^{3}$. Here we will use the added algebraic structures associated to Heegaard-Floer theory to identify further complications in the rational cobordism group, $\Theta_{\mathbb{Q}}^{3}$.

As a simple example, an application of the rational homology cobordism classification of lens spaces [Lisca 2007] implies that for $p$ and $q$ relatively prime, the lens space $L(p q, 1)$ is not $\mathbb{Q}$-homology cobordant to any connected sum $L(p, a) \# L(q, b)$. A simple consequence of the work here is that $L(p q, 1)$ is not $\mathbb{Q}$-homology cobordant to any connected sum $M_{p} \# M_{q}$ where $H_{1}\left(M_{p}\right)=\mathbb{Z}_{p}$

This work was supported in part by the National Science Foundation under Grant 1007196 and by the National Research Foundation of Korea (NRF) grant funded by the Korea government (MEST) NRF-2011-0012893.

MSC2010: primary 57M27; secondary 57M25.

Keywords: three-manifold, connected sum, homology cobordism, knot concordance. 
and $H_{1}\left(M_{q}\right)=\mathbb{Z}_{q}$. We let $\Theta_{R}^{3}$ denote the $R$-homology cobordism group of three-dimensional $R$-homology spheres. Note that $\Theta_{\mathbb{Z}[1 / p]}^{3}$ is generated by threemanifolds $M$ with $H_{1}(M) p$-torsion. There is a canonical map

$$
\Phi: \bigoplus_{p \in \mathscr{P}} \Theta_{\mathbb{Z}[1 / p]}^{3} \rightarrow \Theta_{\mathbb{Q}}^{3}
$$

Rochlin's theorem and Furuta's result imply that the kernel of $\Phi$ is infinitely generated. To simplify notation, we abbreviate the summation by $\bigoplus \Theta_{p}$; our main result is the following:

Proposition. The cokernel of $\Phi, \Theta_{\mathbb{Q}}^{3} / \Phi\left(\bigoplus \Theta_{p}\right)$, contains a free subgroup of infinite rank generated by lens spaces of the form $L(p q, 1)$. It also contains an infinite subgroup generated by elements of order two: lens spaces of the form $L\left(4 n^{2}+1,2 n\right)$. An infinite subgroup is also generated by three-manifolds that bound $\mathbb{Q}$-homology balls topologically.

We also present applications to the study of knot concordance and present families of elements in the kernel $\Theta_{\mathbb{Q}}^{3} / \Theta_{\mathbb{Z}}^{3} \rightarrow \widehat{\Theta}_{\mathbb{Q}}^{3}$, where $\widehat{\Theta}_{\mathbb{Q}}^{3}$ denotes the topological cobordism group. Similar examples were presented in [Hedden et al. 2012], with the additional condition that bordisms were assumed to be spin.

An important perspective is provided by considering the torsion linking form of three-manifolds, which yields a homomorphism $\Theta_{\mathbb{Q}}^{3} \rightarrow W(\mathbb{Q} / \mathbb{Z})$, the Witt group of nondegenerate symmetric $\mathbb{Q} / \mathbb{Z}$-valued bilinear forms on finite abelian groups. According to [Kawauchi and Kojima 1980] this homomorphism is surjective. Again by Rochlin's theorem and Furuta's result, it has infinitely generated kernel (in the topological category it is conjecturally an isomorphism). There is a canonical isomorphism $\bigoplus_{p \in \mathscr{P}} W\left(\mathbb{F}_{p}\right) \rightarrow W(\mathbb{Q} / \mathbb{Z})$, where $W\left(\mathbb{F}_{p}\right)$ is the Witt group of nondegenerate symmetry bilinear forms on $\mathbb{F}_{p}$-vector spaces and $\mathscr{P}$ is the set of prime integers. The conjecture that topological cobordism is determined by the linking form implies that $\widehat{\Theta}_{\mathbb{Q}}^{3}$ has a corresponding primary decomposition. One thrust of our work here is to display the extent of the failure of the existence of such a primary decomposition in the smooth setting.

The commutative diagram in Figure 1 organizes the groups of interest.

The proposition above states that $\Theta_{\mathbb{Q}}^{3} / \operatorname{Image}(\Phi)$ is infinitely generated containing an infinite free subgroup and infinite two-torsion and that, furthermore, the image of $\mathscr{K}$ in $\Theta_{\mathbb{Q}}^{3} /$ Image $(\Phi)$ similarly contains an infinite subgroup.

Definition. A three-manifold $M$ is said to split if it represents a class in the image of $\Phi$. That is, a manifold does not split if it is nontrivial in the cokernel of $\Phi$.

Outline. In Sections 2, 3, and 4 we present some of the basic definitions used throughout the paper, isolate a basic result concerning metabolizers of nondegenerate 


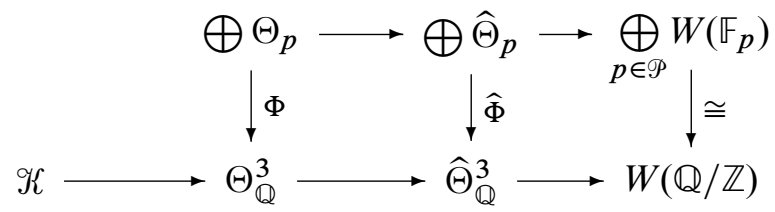

Figure 1. Groups of interest. Hats denote the topological category and $\mathscr{K}$ denotes the kernel of the canonical homomorphism from the smooth to the topological $\mathbb{Q}$-homology cobordism group. With the exception of the inclusion of the kernel, all horizontal arrows are surjective. Conjecturally the right square consists of isomorphisms.

symmetric bilinear forms, and discuss $\operatorname{Spin}^{c}$ structures. Section 5 presents one of our main results, describing an obstruction based on Heegaard-Floer $d$-invariants to a class in $\Theta_{\mathbb{Q}}^{3}$ being in the image $\Phi\left(\bigoplus \Theta_{p}\right)$. Following this we provide a series of examples:

- Section 6 demonstrates that lens spaces $L(p q, 1)$ with $p$ and $q$ square-free and relatively prime do not split, and extends this to finite connected sums of such lens spaces, with all $p$ and $q$ distinct, thus proving that $\Theta_{\mathbb{Q}}^{3} / \Phi\left(\Theta_{p}\right)$ is infinite. Section 7 further extends this, demonstrating that the set of lens spaces of the form $L(p q, 1)$ (with $p$ and $q$ now required to be prime) generate an infinite free subgroup of infinite rank contained in $\Theta_{\mathbb{Q}}^{3} / \Phi\left(\bigoplus \Theta_{p}\right)$.

- Section 8 considers specific lens spaces of the form $L\left(4 n^{2}+1,2 n\right)$ to provide elements of order 2 in $\Theta_{\mathbb{Q}}^{3}$ that do not split, in particular showing that $\Theta_{\mathbb{Q}}^{3} / \Phi\left(\bigoplus \Theta_{p}\right)$ contains 2 -torsion. Section 9 expands on this, providing an infinite family of independent elements of order 2.

- Section 10 begins the examination of the failure of splittings among manifolds that do split topologically; that is, we consider manifolds representing classes in $\mathscr{K}$. The main example is built from surgery on the connected sum of the torus knot $T_{3,5}$ and the untwisted Whitehead double of the trefoil knot, $\mathrm{Wh}\left(T_{2,3}\right)=D$. We show that $S_{15}^{3}\left(T_{3,5} \# D\right)$ splits topologically but not smoothly. Section 11 generalizes that example to an infinite family, using $(p, p+2)$ torus knots, with $p$ odd.

- Section 12 applies the results of Section 6 to demonstrate the failure of a splitting theorem for knot concordance which, by a result of Stoltzfus [1977], applies algebraically and in dimensions greater than 3 .

- According to [Freedman 1982; Freedman and Quinn 1990], all homology spheres bound contractible 4-manifolds topologically, so $\Theta_{\mathbb{Z}}^{3} \subset \mathcal{K}$. In Section 13 
we outline the proof that the quotient $\mathscr{K} / \Theta_{\mathbb{Z}}^{3}$ contains an infinitely generated free subgroup. This was proved in [Hedden et al. 2012] with the added constraint that one restricts the cobordism groups by considering only manifolds that are $\mathbb{Z}_{2}$-homology spheres or by requiring that all spaces have spin structures. We briefly indicate how results here permit one to remove those restrictions in the argument in the same reference.

\section{Definitions}

We will consider $\mathbb{Q}$-homology 3-spheres: these are closed 3-manifolds $M^{3}$ with $H_{n}\left(M^{3}, \mathbb{Q}\right) \cong H_{n}\left(S^{3}, \mathbb{Q}\right)$ for all $n$. For each such $M$ there is a symmetric linking form $\beta: H_{1}(M) \times H_{1}(M) \rightarrow \mathbb{Q} / \mathbb{Z}$ which is nondegenerate in the sense that the induced map $\beta^{*}: H_{1}(M) \rightarrow \operatorname{Hom}\left(H_{1}(M), \mathbb{Q} / \mathbb{Z}\right)$ is an isomorphism. If $M=\partial X^{4}$, where $X$ is a compact 4-manifold and $H_{n}(X, \mathbb{Q})=H_{n}\left(B^{4}, \mathbb{Q}\right)$ for all $n$, then the kernel $M$ of the map $H_{1}(M) \rightarrow H_{1}(X)$ is a metabolizer for $\beta$ (see [Casson and Gordon 1986]). That is, $\mu^{\perp}=M$, and in particular $|M|^{2}=\left|H_{1}(M)\right|$. The Witt group $W(\mathbb{Q} / \mathbb{Z})$ is built from the set of all pairs $(G, \beta)$ where $G$ is a finite abelian group and $\beta$ is a nondegenerate symmetric bilinear form taking values in $\mathbb{Q} / \mathbb{Z}$. There is an equivalence relation on this set: $(G, \beta) \sim\left(G^{\prime}, \beta^{\prime}\right)$ if $\left(G \oplus G^{\prime}, \beta \oplus-\beta^{\prime}\right)$ has a metabolizer, and under this relation it becomes an abelian group under direct sum, denoted $W(\mathbb{Q} / \mathbb{Z})$. It can be proved (for instance, see [Alexander et al. 1976]) that a pair $(G, \beta)$ is Witt trivial if and only if it has a metabolizer. The proof of this fact includes the following, which we will be using.

Proposition 1. If $\left(G_{1}, \beta_{1}\right) \oplus\left(G_{2}, \beta_{2}\right)$ has metabolizer $M$ and $\left(G_{2}, \beta_{2}\right)$ has metabolizer $\mathcal{M}_{2}$, then $\mathcal{M}_{1}=\left\{g \in G_{1} \mid(g, h) \in \mathcal{M}\right.$ for some $\left.h \in \mathcal{M}_{2}\right\}$ is a metabolizer for $\left(G_{1}, \beta_{1}\right)$.

The Witt groups $W(\mathbb{Q} / \mathbb{Z},\langle p\rangle)$ are defined as is $W(\mathbb{Q} / \mathbb{Z})$, considering only $p$-torsion abelian groups, and the decomposition $W(\mathbb{Q} / \mathbb{Z}) \cong \bigoplus_{p \in \mathscr{P}} W(\mathbb{Q} / \mathbb{Z},\langle p\rangle)$ is easily proved. The Witt group of nondegenerate symmetric forms on $\mathbb{F}_{p}$-vector spaces is denoted $W\left(\mathbb{F}_{p}\right)$. The inclusion $W\left(\mathbb{F}_{p}\right) \rightarrow W(\mathbb{Q} / \mathbb{Z},\langle p\rangle)$ is an isomorphism. In the proof of this, the inclusion is clearly injective, and an inverse map $W(\mathbb{Q} / \mathbb{Z},\langle p\rangle) \rightarrow W\left(\mathbb{F}_{p}\right)$ is explicitly constructed via "devissage" [Alexander et al. 1976; Milnor and Husemoller 1973].

Let $R$ be a commutative ring. Two closed 3-manifolds, $M_{1}$ and $M_{2}$, are called $R$-homology cobordant if there is a compact smooth 4-manifold $X$ with boundary the disjoint union $M_{1} \cup-M_{2}$ such that the inclusions $H_{*}\left(M_{i}, R\right) \rightarrow H_{*}(X, R)$ are isomorphisms. Equivalently they are $R$-cobordant, written $M_{1} \sim_{R} M_{2}$, if $M_{1} \#-M_{2}$ bounds an $R$-homology 4-ball. The set of $R$-cobordism classes of $R$-homology spheres forms an abelian group with operation induced by connected sum. This group is denoted $\Theta_{R}^{3}$. 
The ring $\mathbb{Z}[1 / p]$ is the ring of integers with $p$ inverted, consisting of all rational numbers with denominator a power of $p$. A closed 3-manifold $M$ is a $\mathbb{Z}[1 / p]$-homology sphere if and only if $H_{1}(M)$ is $p$-torsion. The linking form provides well-defined homomorphisms $\Theta_{\mathbb{Q}}^{3} \rightarrow W(\mathbb{Q} / \mathbb{Z})$ and $\Theta_{\mathbb{Z}[1 / p]}^{3} \rightarrow W\left(\mathbb{F}_{p}\right)$ for which the following diagram commutes. As in the introduction, we abbreviate $\Theta_{\mathbb{Z}[1 / p]}^{3}$ by $\Theta_{p}$.

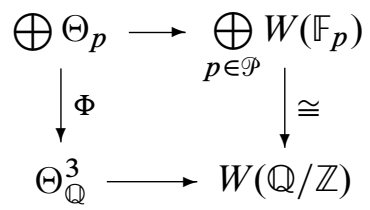

If we switch to the topological category, all these maps are conjecturally isomorphisms.

\section{Metabolizers for connected sums}

3.1. Metabolizers. If a connected sum of 3-manifolds bounds a rational homology ball, the associated metabolizer of the linking form does not necessarily split relative to the connected sum. As a simple example, for the connected sum of lens spaces $L(p, 1) \#-L(p, 1)$ with $p$ prime, the only metabolizers for the linking form on $\mathbb{Z}_{p} \oplus \mathbb{Z}_{p}$ are the diagonal and skew diagonal subgroups, generated by $(1, \pm 1)$. However the existence of the connected sum decomposition does place constraints on the metabolizer.

Theorem 2. If $p$ is prime, $G$ is a finite abelian group, and a given nondegenerate symmetric bilinear form $\beta_{1} \oplus \beta_{2}$ on $\mathbb{Z}_{p} \oplus G$ has metabolizer $\mathcal{M}$, then for some $a \in G,(1, a) \in \mathcal{M}$.

Proof. Let $G_{p}$ denote the $p$-torsion in $G$. There is a metabolizer $M_{p}$ for the form restricted to $\mathbb{Z}_{p} \oplus G_{p}$. If $\mu_{p} \subset G_{p}$, then it would represent a metabolizer for the linking form restricted to $G_{p}$, implying that the order of $G_{p}$ is an even power of $p$. But since the form on $\mathbb{Z}_{p} \oplus G_{p}$ is metabolic, the order of $G_{p}$ must be an odd power of $p$. It follows that there is an element $\left(a^{\prime}, a^{\prime \prime}\right) \in M_{p}$ with $a^{\prime} \neq 0$. Multiplying by $\left(a^{\prime}\right)^{-1} \bmod p$, we see that $(1, a) \in \mathcal{M}_{p} \subset \mathcal{M}$ for some $a \in G_{p}$.

In the following corollary, for each integer $k, G_{k}$ denotes a finite abelian group of order dividing a power of $k$.

Corollary 3. If $m$ is a square-free integer, $G_{m} \oplus G_{n}$ is a finite abelian group with $\operatorname{gcd}(m, n)=1$ and a given nondegenerate symmetric bilinear form $\beta_{1} \oplus \beta_{2} \oplus \beta_{3}$ on $\mathbb{Z}_{m} \oplus G_{m} \oplus G_{n}$ has metabolizer $\mathcal{M}$, then for some $a \in G_{m},(1, a, 0) \in \mathcal{M}$.

Proof. Write $\mathbb{Z}_{m}=\mathbb{Z}_{p_{1}} \oplus \cdots \oplus \mathbb{Z}_{p_{k}}$ with each $p_{i}$ prime. (Since $m$ is square-free, there are no prime powers in the factorization of $m$ and all $p_{i}$ are distinct.) By Theorem 2, 
the projection of $\mathcal{M}$ to each $\mathbb{Z}_{p_{i}}$ summand is surjective. Since the $p_{i}$ are relatively prime, the projection to $\mathbb{Z}_{m}$ is similarly surjective.

In order to construct elements of infinite order, we will need to consider multiples of linking forms. Without loss of generality, we will be able to assume that the multiplicative factors are divisible by four.

Theorem 4. Suppose that $p$ is prime and a nondegenerate symmetric bilinear form $4 k\left(\beta_{1} \oplus \beta_{2}\right)$ on $\left(\mathbb{Z}_{p} \oplus G\right)^{4 k}$ has a metabolizer $\mathcal{M}$. Then $M$ contains an element of the form $\left(1,1, \ldots, 1, \alpha_{2 k+1}, \ldots, \alpha_{4 k}\right) \oplus b$ for some set of $\alpha_{i} \in \mathbb{Z}_{p}$ and some $b \in G^{4 k}$.

Proof. The Witt group $W(\mathbb{Q} / \mathbb{Z})$ is 4-torsion [Milnor and Husemoller 1973], and thus $4 k \beta_{2}$ has a metabolizer $\mathcal{M}^{\prime}$. By Proposition 1 , the set of elements $x$ such that $(x, y) \in \mathcal{M}$ for some $y \in \mathcal{M}^{\prime}$ is a metabolizer, denoted $\mathcal{N}$, for $4 k \beta_{1}$, and thus is $2 k$-dimensional. As argued in [Livingston and Naik 1999], a simple application of the Gauss-Jordan algorithm applied to a generating set for $\mathcal{N}$ yields a generating set consisting of vectors of the form $(1,0,0,0, \ldots, 0, *, *, \ldots)$, $(0,1,0,0, \ldots, 0, *, *, \ldots),(0,0,1,0, \ldots, 0, *, *, \ldots), \ldots$, where each initial sequence of a 1 and 0 s is of length $2 k$.

By adding these vectors together, we find that the metabolizer $\mathcal{N}$ contains an element of the form $\left(1,1, \ldots, 1, \alpha_{2 k+1}, \ldots, \alpha_{4 k}\right) \in \mathbb{Z}_{p}^{4 k}$. Finally, since each element in $\mathcal{N}$ pairs with an element in the metabolizer $\mathcal{M}^{\prime}$ to give an element in $\mathcal{M}$, we get the desired element $b$.

\section{Spin ${ }^{c}$ structures}

We need the following facts about $\operatorname{Spin}^{c}(Y)$, the set of $\operatorname{Spin}^{c}$ structures on manifolds.

- The first Chern class is a map $c_{1}: \operatorname{Spin}^{c}(Y) \rightarrow H^{2}(Y)$.

- There is a transitive action $H^{2}(Y) \times \operatorname{Spin}^{c}(Y) \rightarrow \operatorname{Spin}^{c}(Y)$ denoted $(\alpha, \mathfrak{s}) \rightarrow \alpha \cdot \mathfrak{s}$.

- For $Y \subset W$ a codimension-one submanifold with trivial normal bundle, such as a boundary component of $W$, the restriction map $r$ is functorial: If $\mathfrak{s} \in$ $\operatorname{Spin}^{c}(W), \alpha \in H^{2}(W)$, then

$$
r(\alpha \cdot \mathfrak{s})=r(\alpha) \cdot r(\mathfrak{s}) .
$$

- For all $\alpha \in H^{2}(Y)$ and $\mathfrak{s} \in \operatorname{Spin}^{c}(Y), c_{1}(\alpha \cdot \mathfrak{s})-c_{1}(\mathfrak{s})=2 \alpha$.

- As a corollary, if $\left|H^{2}(Y)\right|$ is finite and odd, then $c_{1}: \operatorname{Spin}^{c}(Y) \rightarrow H^{2}(Y)$ is a bijection.

- There is a canonical bijection $\operatorname{Spin}^{c}\left(Y_{1} \# Y_{2}\right) \rightarrow \operatorname{Spin}^{c}\left(Y_{1}\right) \times \operatorname{Spin}^{c}\left(Y_{2}\right)$. 
For every smooth 4-manifold $W$, the set $\operatorname{Spin}^{c}(W)$ is nonempty. (See [Gompf and Stipsicz 1999] for a proof.) As a consequence, we have the following.

Theorem 5. Let $N=\partial X$ and let $\mathfrak{s} \in \operatorname{Spin}^{c}(N)$ be the restriction of a $\operatorname{Spin}^{c}$ structure on $X$. Then the $\operatorname{Spin}^{c}$ structures on $N$ which extend to $X$ are those of the form $\alpha \cdot \mathfrak{s}$ for $\alpha$ in the image of the restriction map $r: H^{2}(X) \rightarrow H^{2}(N)$.

4.1. Identifying $\boldsymbol{H}_{1}(N)$ and $\boldsymbol{H}^{2}(N)$. Let $N$ be a rational homology 3-sphere bounding a rational homology ball $X$. Then, by Poincaré duality, $H_{1}(N) \cong H^{2}(N)$. We have denoted the kernel of $H_{1}(N) \rightarrow H_{1}(X)$ by $M$. Via duality, it corresponds to the image of $H^{2}(X)$ in $H^{2}(N)$. Thus we will use $M$ to denote this subgroup of $H^{2}(N)$.

4.2. Spin structures. If the order $\left|H_{1}(M)\right|$ is odd, then there is a unique spin structure on $M$ that lifts to a canonical $\operatorname{Spin}^{c}$ structure that we will denote $\mathfrak{s}_{0}$. With this, there is a natural identification of $H^{2}(M)$ with $\operatorname{Spin}^{c}(M)$. However we face the complication that in assuming that $M$ bounds a rational homology 4-ball $X$, we cannot assume that $X$ has a spin structure. The following result permits us to adapt to this possibility. (In addition to playing a role in considering splittings of classes in $\Theta_{\mathbb{Q}}^{3}$, in Section 13 we will use this result to extend a theorem from [Hedden et al. 2012] in which an added hypothesis was needed to ensure the existence of a spin structure on $X$.)

Theorem 6. Suppose that $N_{1} \# N_{2}=\partial X$ for some smooth rational homology 4-ball $X$ and that the order of $H_{1}\left(N_{1}\right)$ is odd. Then the image of the restriction map $\operatorname{Spin}^{c}(X) \rightarrow \operatorname{Spin}^{c}\left(N_{1}\right)$ contains the spin structure $\mathfrak{s}_{0} \in \operatorname{Spin}^{c}\left(N_{1}\right)$. In particular, every element in the image of this restriction map is of the form $\alpha \cdot \mathfrak{s}_{0}$ for $\alpha \in$ Image $\left(H^{2}(X) \rightarrow H^{2}\left(N_{1}\right)\right)$.

Proof. Let $H=\operatorname{Image}\left(H^{2}(X) \rightarrow H^{2}\left(N_{1}\right)\right), S=\operatorname{Image}\left(\operatorname{Spin}^{c}(X) \rightarrow \operatorname{Spin}^{c}\left(N_{1}\right)\right)$. As usual, the choice of an element $\mathfrak{s} \in S$ determines a bijection between $H$ and $S$. In particular, the number of elements in $S$ is the same as in $H$, which is odd. Conjugation defines an involution on $S$ which commutes with restriction. Thus since $S$ is odd, conjugation has a fixed point in $S$. But the only fixed element under conjugation is the spin structure, since $c_{1}(\overline{\mathfrak{s}})=-c_{1}(\mathfrak{s})$.

\section{Basic obstructions from $d$-invariants}

To each rational homology 3-sphere $M$ and $\mathfrak{s} \in \operatorname{Spin}^{c}(M)$ there is associated an invariant $d(M, \mathfrak{s}) \in \mathbb{Q}$, defined in [Ozsváth and Szabó 2003]. It is additive under connected sum: $d\left(M \# N,\left(\mathfrak{s}_{1}, \mathfrak{s}_{2}\right)\right)=d\left(M, \mathfrak{s}_{1}\right)+d\left(N, \mathfrak{s}_{2}\right)$. A key result relating the $d$-invariant and bordism is the following, taken from the same reference.

Theorem 7. If $M=\partial X$ with $H_{*}(X, \mathbb{Q}) \cong H_{*}\left(B^{4}, \mathbb{Q}\right)$, and $\mathfrak{t} \in \operatorname{Spin}^{c}(X)$, then $d\left(M,\left.\mathfrak{t}\right|_{M}\right)=0$. 
5.1. Obstruction theorem. Suppose that $\left|H_{1}(M)\right|$ is odd and $\mathfrak{s}_{0}$ is the unique spin structure on $M$. For $\alpha \in H^{2}(M)$, we abbreviate $d\left(M, \alpha \cdot \mathfrak{s}_{0}\right)$ by $d(M, \alpha)$.

Definition 8. $\bar{d}(M, \alpha)=d(M, \alpha)-d(M, 0)$.

The following result will be sufficient to prove that $\Theta_{\mathbb{Q}}^{3} / \Phi\left(\bigoplus \Theta_{p}\right)$ is infinite.

Theorem 9. Suppose $\left\{M_{i}\right\}$ is a set of 3-manifolds for which $H_{1}\left(M_{i}\right)=\mathbb{Z}_{m_{i}} \oplus \mathbb{Z}_{n_{i}}$, where the $m_{i}$ and $n_{i}$ are square-free and odd, and the elements of the full set $\left\{m_{i}, n_{i}\right\}$ are pairwise relatively prime. If a finite connected sum $\#_{k=1}^{N} \pm M_{i_{k}}$ represents a class in $\Theta_{\mathbb{Q}}^{3}$ that is in the image $\Phi\left(\bigoplus \Theta_{p}\right)$, then for all $i=i_{k}, 1 \leq k \leq N$, and for all $(a, b) \in \mathbb{Z}_{m_{i}} \oplus \mathbb{Z}_{n_{i}}$,

$$
\bar{d}\left(M_{i},(a, b)\right)=\bar{d}\left(M_{i},(a, 0)\right)+\bar{d}\left(M_{i},(0, b)\right) .
$$

Proof. Let $Y=\#_{k} \pm M_{i_{k}}$. We consider $k=1$, abbreviating $M_{i_{1}}=M$ and $H_{1}(M) \cong \mathbb{Z}_{m} \oplus \mathbb{Z}_{n}$. Suppose that $Y$ is in the image of $\Phi$. Then $Y \# \oplus Y_{p_{i}}=\partial X$ for some collection of $\mathbb{Z}\left[p_{i}^{-1}\right]$-homology spheres $Y_{p_{i}}$ and a rational homology ball $X$. Collecting summands, we can write $M \# N_{m} \# N_{n} \# N=\partial X$, where the prime factors of $\left|H_{1}\left(N_{m}\right)\right|$ all divide $m$, the prime factors of $\left|H_{1}\left(N_{n}\right)\right|$ all divide $n$, and $\left|H_{1}(N)\right|$ is relatively prime to $m n$. Let $\left(\mathfrak{s}_{0}, \mathfrak{s}_{1}, \mathfrak{s}_{2}, \mathfrak{s}_{*}\right) \in \operatorname{Image}\left(\operatorname{Spin}^{c}(X)\right)$. (By Theorem 6 we can assume that the structure $\mathfrak{s}_{0} \in \operatorname{Spin}^{c}(M)$ is the spin structure.) Then, by Corollary 3 , for all $a \in \mathbb{Z}_{m}$ and $b \in \mathbb{Z}_{n}$, there are elements $a^{\prime} \in H_{1}\left(N_{m}\right)$ and $b^{\prime} \in H_{1}\left(N_{n}\right)$ such that:

- $\left((a, 0) \cdot \mathfrak{s}_{0}, a^{\prime} \cdot \mathfrak{s}_{1}, \mathfrak{s}_{2}, \mathfrak{s}_{*}\right) \in \operatorname{Image}\left(\operatorname{Spin}^{c}(X)\right)$.

- $\left((0, b) \cdot \mathfrak{s}_{0}, \mathfrak{s}_{1}, b^{\prime} \cdot \mathfrak{s}_{2}, \mathfrak{s}_{*}\right) \in \operatorname{Image}\left(\operatorname{Spin}^{c}(X)\right)$.

- $\left((a, b) \cdot \mathfrak{s}_{0}, a^{\prime} \cdot \mathfrak{s}_{1}, b^{\prime} \cdot \mathfrak{s}_{2}, \mathfrak{s}_{*}\right) \in \operatorname{Image}\left(\operatorname{Spin}^{c}(X)\right)$.

Thus we have the following vanishing conditions on the $d$-invariants:

- $d\left(M, \mathfrak{s}_{0}\right)+d\left(N_{m}, \mathfrak{s}_{1}\right)+d\left(N_{n}, \mathfrak{s}_{2}\right)+d\left(N, \mathfrak{s}_{*}\right)=0$.

- $d\left(M,(a, 0) \cdot \mathfrak{s}_{0}\right)+d\left(N_{m}, a^{\prime} \cdot \mathfrak{s}_{1}\right)+d\left(N_{n}, \mathfrak{s}_{2}\right)+d\left(N, \mathfrak{s}_{*}\right)=0$.

- $d\left(M,(0, b) \cdot \mathfrak{s}_{0}\right)+d\left(N_{m}, \mathfrak{s}_{1}\right)+d\left(N_{n}, b^{\prime} \cdot \mathfrak{s}_{2}\right)+d\left(N, \mathfrak{s}_{*}\right)=0$.

- $d\left(M,(a, b) \cdot \mathfrak{s}_{0}\right)+d\left(N_{m}, a^{\prime} \cdot \mathfrak{s}_{1}\right)+d\left(N_{n}, b^{\prime} \cdot \mathfrak{s}_{2}\right)+d\left(N, \mathfrak{s}_{*}\right)=0$.

Subtracting the second and third equality from the sum of the first and fourth yields

$$
d\left(M,(a, b) \cdot \mathfrak{s}_{0}\right)-d\left(M,(a, 0) \cdot \mathfrak{s}_{0}\right)-d\left(M,(0, b) \cdot \mathfrak{s}_{0}\right)+d\left(M, \mathfrak{s}_{0}\right)=0 .
$$

Recalling that $\bar{d}(M, \alpha)$ denotes $d\left(M, \alpha \cdot \mathfrak{s}_{0}\right)-d\left(M, \mathfrak{s}_{0}\right)$, this can be rewritten as

$$
\bar{d}(M,(a, b))-\bar{d}(M,(a, 0))-\bar{d}(M,(0, b))=0 .
$$

Repeating for each $M_{i}$ completes the proof of the theorem. 


\section{Lens space examples: $L(p q, 1)$.}

Let $\left(p_{i}, q_{i}\right)$ be pairs of relatively prime square-free odd integers such the products $p_{i} q_{i}$ are pairwise relatively prime. We prove:

Theorem 10. No finite linear combination $\#_{k} \pm L\left(p_{i_{k}} q_{i_{k}}, 1\right)$ represents an element in the image $\Phi\left(\bigoplus \Theta_{p}\right) \subset \Theta_{\mathbb{Q}}^{3}$.

Proof. To simplify notation, we use $L_{n}$ to denote $L(n, 1)$. Assume there is such a finite linear combination. We consider the first term $L_{p_{1} q_{1}}$ and simplify notation by writing $p=p_{1}$ and $q=q_{1}$. By Theorem 9 we would have for all $(a, b) \in \mathbb{Z}_{p} \oplus \mathbb{Z}_{q}$,

$$
\bar{d}\left(L_{p q},(a, b)\right)=\bar{d}\left(L_{p q},(a, 0)\right)+\bar{d}\left(L_{p q},(0, b)\right) .
$$

According to [Ozsváth and Szabó 2003], for some enumeration of $\operatorname{Spin}^{c}$ structures on $L(m, n)$, denoted $\mathfrak{s}_{i}, 0 \leq i<m$, if we let $D(m, n, i)=d\left(-L(m, n), \mathfrak{s}_{i}\right)$, there is the recursive formula

$$
D(m, n, i)=\frac{m n-(2 i+1-m-n)^{2}}{4 m n}-D\left(n, m^{\prime}, i^{\prime}\right),
$$

where the primes denote reductions modulo $n, 0<n<m$, and $0 \leq i<m$. The base case in the recursion is by definition $D(1,0,0)=0$. For every $\operatorname{Spin}^{c}$ structure $\mathfrak{s}$ there is a conjugate structure $\overline{\mathfrak{s}}$ for which $d(M, \mathfrak{s})=d(M, \overline{\mathfrak{s}})$ and $\mathfrak{s} \neq \overline{\mathfrak{s}}$ unless $\mathfrak{s}$ is the spin structure. We claim that for $L_{p q}$, the $\operatorname{Spin}^{c}$ structure $\mathfrak{s}_{0}$ does correspond to the spin structure. Indeed, an algebraic computation shows that $4 p q D(p q, 1, i)=$ $-4 i^{2}+4 p q i+p q(1-p q)$, and in particular, $p q D(p q, 1,0)=p q(1-p q)$. The difference,

$$
4 p q D(p q, 1, i)-4 p q D(p q, 1,0)=4 i(p q-i),
$$

does not take on the value 0 for any $0<i<p q$. Since the value of $D(p q, 1,0)$ is unique among the $d$-invariants, it must correspond to the spin structure. In applying Theorem 9 , we identify $\mathbb{Z}_{p} \oplus \mathbb{Z}_{q} \cong \mathbb{Z}_{p q}$, so that the pair $(a, b) \in \mathbb{Z}_{p} \oplus \mathbb{Z}_{q}$ corresponds to $a q+b p \in \mathbb{Z}_{p q}$. In this case, the criterion becomes

$$
D(p q, 1, a p+b q)-D(p q, 1, a p)-D(p q, 1, b q)+D(p q, 1,0)=0 .
$$

Certainly $p+q<p q$, so we can apply the formula for $D$ with $a=b=1$. However, in this case, the sum is immediately calculated to equal -2 , which is not 0 .

\section{Infinite order examples}

The examples of the previous section are sufficient to demonstrate that the quotient $\Theta_{\mathbb{Q}}^{3} / \Phi\left(\bigoplus \Theta_{p}\right)$ is infinite. We now present an argument to show it contains an infinitely generated free subgroup. To carry out this argument we need to make the additional assumption of primeness for the relevant $p$ and $q$. Let $\left\{p_{i}, q_{i}\right\}$ be a 
set of distinct odd prime pairs with all elements distinct. We continue to denote $L(n, 1)$ by $L_{n}$. This section is devoted to the proof of the following theorem.

Theorem 11. The lens spaces $L_{p_{i} q_{i}}$ are linearly independent in the quotient group $\Theta_{\mathbb{Q}}^{3} / \Phi\left(\bigoplus \Theta_{p}\right)$.

7.1. Notation. Suppose that $\sum_{i} b_{i} L_{p_{i} q_{i}} \subset$ Image $(\Phi)$. We can assume that $b_{1} \neq 0$. We simplify notation, writing $p$ and $q$ for $p_{1}$ and $q_{1}$, respectively. There is no loss of generality in assuming that for all $i, b_{i}=4 k_{i}$ for some $k_{i}$, and write $k=k_{1}$.

Following our earlier approach, we will show that a contradiction arises from the assumption that $N=4 k L_{p q} \# M_{p} \# M_{q} \# M_{*}=\partial X$ for some rational homology 4-ball $X$, where the orders of $H_{1}\left(M_{p}\right)$ and $H_{1}\left(M_{q}\right)$ are powers of $p$ and $q$, respectively, and the order of $H_{1}\left(M_{*}\right)$ is relatively prime to $p q$.

According to Theorem 4 , the $p$-primary part of the associated metabolizer, $\mu_{p}$, includes a vector $A=\left(\left(1, \ldots, 1, \alpha_{2 k+1}, \ldots, \alpha_{4 k}\right), g\right) \in\left(\mathbb{Z}_{p}\right)^{4 k} \oplus H_{1}\left(M_{p}\right)$. Similarly the $q$-primary part of the associated metabolizer, $M_{q}$, includes a vector $B=\left(\left(1, \ldots, 1, \beta_{2 k+1}, \ldots, \beta_{4 k}\right), h\right) \in\left(\mathbb{Z}_{q}\right)^{4 k} \oplus H_{1}\left(M_{q}\right)$.

7.2. Constraints on the d-invariants. We let the spin structures on $L(p q, 1), M_{p}$, and $M_{q}$ be $\mathfrak{s}_{0}, \mathfrak{s}_{0}^{\prime}$ and $\mathfrak{s}_{0}^{\prime \prime}$, respectively. Consider now the vectors $0, a A, b B$, and $a A+b B \in \mathcal{M}$. Computing the $d$-invariant associated to each, we find that each of the following sums is 0 :

$2 k d\left(L_{p q}, s_{0}\right)+\sum_{i=2 k+1}^{4 k} d\left(L_{p q}, \mathfrak{s}_{0}\right)+d\left(M_{p}, \mathfrak{s}_{0}^{\prime}\right)+d\left(M_{q}, \mathfrak{s}_{0}^{\prime \prime}\right)+d\left(M_{*}, \mathfrak{t}\right)$,
$2 k d\left(L_{p q}, a q \cdot s_{0}\right)+\sum_{i=2 k+1}^{4 k} d\left(L_{p q}, a q \alpha_{i} \cdot \mathfrak{s}_{0}\right)+d\left(M_{p}, a g \cdot \mathfrak{s}_{0}^{\prime}\right)+d\left(M_{q}, \mathfrak{s}_{0}^{\prime \prime}\right)+d\left(M_{*}, \mathfrak{t}\right)$, $2 k d\left(L_{p q}, b p \cdot s_{0}\right)+\sum_{i=2 k+1}^{4 k} d\left(L_{p q}, b p \beta_{i} \cdot \mathfrak{s}_{0}\right)+d\left(M_{p}, \mathfrak{s}_{0}^{\prime}\right)+d\left(M_{q}, b h \cdot \mathfrak{s}_{0}^{\prime \prime}\right)+d\left(M_{*}, \mathfrak{t}\right)$, $2 k d\left(L_{p q},(a q+b p) \cdot s_{0}\right)+\sum_{i=2 k+1}^{4 k} d\left(L_{p q},\left(a q \alpha_{i}+b p \beta_{i}\right) \cdot \mathfrak{s}_{0}\right)+d\left(M_{p}, a g \cdot \mathfrak{s}_{0}^{\prime}\right)$ $+d\left(M_{q}, b h \cdot \mathfrak{s}_{0}^{\prime \prime}\right)+d\left(M_{*}, \mathfrak{t}\right)$.

Note. We have again used that the inclusion $\mathbb{Z}_{p} \subset \mathbb{Z}_{p q}$ takes $\alpha$ to $\alpha q$, and similarly for $\mathbb{Z}_{q}$ and $\beta$. We now take the sum of the first and last equation, and subtract the sum of the middle two. As before, we continue abbreviating $d\left(M, a \cdot s_{0}\right)$ by $d(M, a)$. The result is that, for some set of $a_{i}$ and $b_{i}$,

$$
\begin{aligned}
& 2 k\left(d\left(L_{p q}, a q+b p\right)-d\left(L_{p q}, a q\right)-d\left(L_{p q}, b p\right)+d\left(L_{p q}, 0\right)\right) \\
& \quad+\sum_{i=2 k+1}^{4 k}\left(d\left(L_{p q}, a_{i} q+b_{i} p\right)-d\left(L_{p q}, a_{i} q\right)-d\left(L_{p q}, b_{i} p\right)+d\left(L_{p q}, 0\right)\right)=0 .
\end{aligned}
$$


We now introduce further notation. Let

$$
\delta\left(L_{p q}, a, b\right)=d\left(L_{p q}, a q+b p\right)-d\left(L_{p q}, a q\right)-d\left(L_{p q}, b p\right)+d\left(L_{p q}, 0\right) .
$$

With this, we have proved the following lemma.

Lemma 12. If the lens spaces $L_{p_{i} q_{i}}$ are linearly dependent in $\Theta_{\mathbb{Q}}^{3} / \Phi\left(\bigoplus \Theta_{p}^{3}\right)$, and, for $p=p_{1}$ and $q=q_{1}, L_{p q}$ has nonzero coefficient in some linear relation, then for all $a$ and $b$ there are $k, a_{i}$ and $b_{i}$ such that

$$
2 k \delta\left(L_{p q}, a, b\right)+\sum_{i=2 k+1}^{4 k} \delta\left(L_{p q}, a_{i}, b_{i}\right)=0 .
$$

7.3. Computation of bounds on $\delta\left(L_{\boldsymbol{p q}}, \boldsymbol{a}, \boldsymbol{b}\right)$. Note that $\delta\left(L_{p q}, a, b\right)=0$ if $a=0$ or $b=0$. Given Lemma 12, the proof of Theorem 11 is completed with the following result.

Lemma 13. For all $a \neq 0 \bmod p$ and $b \neq 0 \bmod q, \delta\left(L_{p q}, a, b\right)<0$.

Proof. All Spin ${ }^{c}$ structures are included by considering the range

$$
-(p-1) / 2 \leq a \leq(p-1) / 2 \text { and }-(q-1) / 2 \leq b \leq(q-1) / 2 .
$$

By symmetry we can exclude the case $a<0$. Since the formula for the $d$-invariant $d\left(L_{p q}, i\right)$ assumes $i \geq 0$, there are three cases to consider.

(1) $a>0, b>0$.

(2) $a>0,-a q / p<b<0$.

(3) $a>0, b<-a q / p$.

The formula for the $d$-invariant in the current case is

$$
4 n\left(d\left(L_{n}, i\right)\right)=n-(2 i+1-n-1)^{2}=n-n^{2}+4 n i-4 i^{2}
$$

for $0 \leq i<n$. We now compute $4 p q \delta\left(L_{p q}, a q+b p\right)$ in each of the three cases. First note that

$$
\delta\left(L_{p q}, a q+b p\right)=d\left(L_{p q}, a q+b p\right)-d\left(L_{p q}, a q\right)-d\left(L_{p q}, b p\right)+d\left(L_{p q}, 0\right) .
$$

Applying the formula for the $d$-invariant, taking care that $i$ is positive in the calculation of $d\left(L_{n}, i\right)$ we find in the first case, $\delta=-8 a b p q$, which is negative. In the second case we compute $\delta=-8 b(a-p) p q$, which is again negative (since $b<0$ and $a<(p-1) / 2)$. In the third case, $\delta=-8 a p q(b+q)$, which is negative since $b>-(q-1) / 2$. This completes the proof. 


\begin{tabular}{r|rrrrrrr}
$a$ & $b=0$ & 1 & 2 & 3 & 4 & 5 & 6 \\
\hline 2 & -52 & -112 & -32 & -72 & 28 & 8 & -2 \\
1 & 52 & -8 & -58 & 32 & -128 & 18 & -28 \\
0 & 0 & 70 & 20 & -20 & 80 & -70 & -80 \\
-1 & 52 & -8 & 72 & 32 & 2 & 112 & -28 \\
-2 & -52 & 18 & -32 & 58 & 28 & 8 & 128 \\
\hline
\end{tabular}

Table 1. $65 d(L(65,8), 13 a+5 b)$.

\begin{tabular}{r|rrrrrrr}
$a$ & $b=0$ & 1 & 2 & 3 & 4 & 5 & 6 \\
\hline 2 & 0 & -2 & 0 & 0 & 0 & 2 & 2 \\
1 & 0 & -2 & -2 & 0 & -4 & 0 & 0 \\
0 & 0 & 0 & 0 & 0 & 0 & 0 & 0 \\
-1 & 0 & -2 & 0 & 0 & -2 & 2 & 0 \\
-2 & 0 & 0 & 0 & 2 & 0 & 2 & 4 \\
\hline
\end{tabular}

Table 2. $d(L(65,8), 13 a+5 b)-d(L(65,8), 13 a)-d(L(65,8), 5 b)$.

\section{An order-2 lens space that does not split}

We now consider a lens space that represents 2-torsion in $\Theta_{\mathbb{Q}}^{3}$. Let $M=L(65,8)$; since $8^{2}=-1 \bmod 65$, we have $M=-M$ and $2 M=0 \in \Theta_{\mathbb{Q}}^{3}$. We show that $M$ does not split. It follows quickly from the fact that $L(65,8)$ is of finite order in $\Theta_{\mathbb{Q}}^{3}$ that, for the spin structure $\mathfrak{s}^{*}, d\left(L(65,8), \mathfrak{s}^{*}\right)=0$. One can compute directly from the formula for $D$ given above that the value 0 is realized only by $\mathfrak{s}_{36}$. Thus, in applying Theorem 9, we identify the homology class $x \in H_{1}(L(65,8))$ with the $\operatorname{Spin}^{c}$ structure $\mathfrak{s}_{36+x}$, where the index is taken modulo 65 . Table 1 presents the values of $d(L(65,8), 13 a+5 b)$ (multiplied by 65 to clear denominators). Rows correspond to the values of $a$ and columns to $b$. The central row and left column correspond to $a=0$ and $b=0$, respectively. Symmetry permits us to list only the values with $b \geq 0$. In Table 2 we list the differences, $d(L(65,8), 13 a+5 b)-$ $d(L(65,8), 13 a)-d(L(65,8), 5 b)$, with the nonzero entries demonstrating the failure of additivity.

\section{Infinite 2-torsion}

We now generalize the previous example to describe an infinite subgroup of $\Theta_{\mathbb{Q}}^{3}$ consisting of 2-torsion that injects into the quotient $\Theta_{\mathbb{Q}}^{3} / \Phi\left(\bigoplus \Theta_{p}\right)$. Consider the family $N_{n}=L\left(4(5 n+1)^{2}+1,2(5 n+1)\right)$; for $n=-1$ we have $-L(65,8)$ as in the previous section, but we simplify the computations by restricting to $n>0$. Expanding, we have $N_{n}=L\left(5\left(20 n^{2}+8 n+1\right), 2(5 n+1)\right)$. If $n \neq 3 \bmod 5$, then 
$20 n^{2}+8 n+1$ is not divisible by 5 . By Appendix A we can further assume that the number $n$ are selected so that $n$ is divisible by 5 , and the integers $20 n^{2}+8 n+1$ are pairwise relatively prime and square-free. We enumerate the set of such $n$ as $n_{i}$ and abbreviate the corresponding lens spaces as $L\left(5 p_{i}, q_{i}\right)=N_{n_{i}}$. The remainder of this section is devoted to proving the following.

Theorem 14. The set $\left\{N_{n_{i}}\right\}$ generates an infinite subgroup consisting of elements of order 2 in $\Theta_{\mathbb{Q}}^{3} / \Phi\left(\bigoplus \Theta_{p}\right)$.

To begin, we need to identify the spin structure. We use the recursion formula

$$
D(m, n, i)=\frac{m n-(2 i+1-m-n)^{2}}{4 m n}-D\left(n, m^{\prime}, i^{\prime}\right)
$$

to compute relevant $d$-invariants. We are interested in the lens spaces $L\left(4 r^{2}+1,2 r\right)$. One step of the recursion reduces this to $L(2 r, 1)$, and another step reduces it to $S^{3}$. Since we need to reduce modulo $2 r$, for $0 \leq i<4 r^{2}+1$, let $y$ be the remainder of $i$ modulo $2 r$ and $x$ the quotient so that $2 r x+y=i$. So we write $\operatorname{Spin}^{c}$ structures as $\mathfrak{s}_{2 r x+y}$ for $0 \leq y<2 r$ and $0 \leq 2 r x+y<4 r^{2}+1$. Carrying out the arithmetic yields:

Lemma 15. For any $r>0, x$ and $y$ with $0 \leq y<2 r$ and $0 \leq 2 r x+y<4 r^{2}+1$ :

(1) $d\left(L\left(4 r^{2}+1,2 r\right), \mathfrak{s}_{2} r x+y\right)=\frac{2\left(r x^{2}+(y-r(2 r+1)) x-r\left(y^{2}-(2 r-1) y-r\right)\right)}{4 r^{2}+1}$.

(2) The discriminant of the numerator, viewed as a quadratic polynomial in the variable $x$, is $4(y-r)^{2}\left(4 r^{2}+1\right)$. Moreover it is the square of an integer if and only if $y=r$.

(3) $d\left(L\left(4 r^{2}+1,2 r\right), \mathfrak{s}_{2 r x+y}\right)=0$ if and only if $x=r$ and $y=r$.

(4) The spin structure on $L\left(4 r^{2}+1,2 r\right)$ is $\mathfrak{s}_{2 r^{2}+r}$.

In our case $r=5 n+1$ and the spin structure is $\mathfrak{s}_{50 n^{2}+25 n+3}$.

Proof of Theorem 14. For each $n$, we write $N_{n}=L\left(5 p_{n}, q_{n}\right)$ and assume that some linear combination $\sum N_{n_{i}}=0 \in \Theta_{\mathbb{Q}}^{3} / \Phi\left(\bigoplus_{p \in \mathscr{P}} \Theta_{\mathbb{Z}[1 / p]}^{3}\right)$. We write the first term in the sum as $N=L(5 p, q)$, where $p=20 n^{2}+8 n+1$. Since the sum splits, for some collection of primes $r_{j}$ and manifolds $M_{r_{j}}$ with $H_{1}\left(M_{r_{j}}\right) r_{j}$-torsion, we have

$$
N \# \underset{i>1}{\#} N_{n_{i}} \# \underset{j}{\#} M_{r_{j}}=\partial X,
$$

where $X$ is a rational homology ball. We can collect terms as $N \# M_{p} \# M_{m}=\partial X$ where $M_{p}$ includes all the $M_{r_{j}}$ for which $r_{j}$ divides $p$, and $M_{m}$ contains all the other summands, including all the $N_{n_{i}}$ with $i>1$.

The homology of this connected sum of three-manifolds splits into the direct sum of three groups: $\left(\mathbb{Z}_{5} \oplus \mathbb{Z}_{p}\right) \oplus G_{p} \oplus G_{m}$, where the order of $G_{p}$ is a product of prime 
factors of $p, 5$ does not divide the order of $G_{p}$, and the orders of $G_{p}$ and $G_{m}$ are relatively prime. It follows that the 5-torsion in the metabolizer, $M_{5}$, is contained in $\left(\mathbb{Z}_{5}, 0\right) \oplus 0 \oplus G_{m}$. The direct sum of all primary parts of the metabolizer for primes that divide $p, \mu_{p}$, is contained in $\mu_{p}=\left(0, \mathbb{Z}_{p}\right) \oplus G_{p} \oplus 0$.

As in our previous arguments, $\mu_{5}$ contains an element of the form $(1,0) \oplus 0 \oplus a^{\prime \prime}$, and $\mathcal{M}_{p}$ contains an element $(0,1) \oplus b^{\prime \prime} \oplus 0$. Continuing as in the early proofs, we find that for all $a$ and $b$,

$$
\bar{d}(L(5 p, q),(a, b))=\bar{d}(L(5 p, q),(a, 0))+\bar{d}(L(5 p, q),(0, b)) .
$$

Or, writing $\mathbb{Z}_{5} \oplus \mathbb{Z}_{p}$ as $\mathbb{Z}_{5 p}$,

$$
\bar{d}(L(5 p, q), p a+5 b)=\bar{d}(L(5 p, q), p a)+\bar{d}(L(5 p, q), 5 b) .
$$

Since $L(5 p, q)$ is of order two, for the spin structure, the $d$-invariant vanishes so the $\bar{d}$-invariant is the same as the $d$-invariant. We let $a=1$ and $b=-1$ and arrive at a contradiction by showing the following equality does not hold:

$$
d(L(5 p, q), p-5)=d(L(5 p, q), p)+d(L(5 p, q),-5) .
$$

To apply Lemma 15 we need to express each of

$$
\left(50 n^{2}+25 n+3\right)+p-5, \quad\left(50 n^{2}+25 n+3\right)+p \quad \text { and } \quad\left(50 n^{2}+25 n+3\right)-5
$$

as $2(5 n+1) x+y$. Simple algebra yields the following pairs $(x, y)$ for these three respective $\operatorname{Spin}^{c}$ structures:

- $a=1, b=-1: \quad(x, y)=(7 n+1,9 n-3)$.

- $a=1, b=0: \quad(x, y)=(7 n+1,9 n+2)$.

- $a=0, b=-1: \quad(x, y)=(5 n+1,5 n-4)$.

Finally one uses these expressions to determine that for all $n$

$$
d(L(5 p, q), p-5)-d(L(5 p, q), p)-d(L(5 p, q),-5)=4 .
$$

Since the difference is not zero, no splitting exists and the proof of Theorem 14 is complete.

\section{Topologically split examples}

In this section, we apply Theorem 9 to find examples of manifolds that split topologically but not smoothly. We begin by carefully examining an example in which the splitting exists smoothly, focusing on the computation of the $d$-invariants and next illustrate the modifications which do not change its topological cobordism class but alter it smoothly. The deepest aspect of the work is in the determination of 
the $d$-invariants. In brief, the manifold we look at is 15 -surgery on the $(3,5)$-torus knot, $T_{3,5}$, denoted by $S_{15}^{3}\left(T_{3,5}\right)$. This is homeomorphic to the connected sum $L(3,5) \#-L(5,3)$. Next, letting $D$ denote the untwisted double of the trefoil knot $\left(D=\mathrm{Wh}\left(T_{2,3}\right)\right)$, which is topologically slice, we consider $S_{15}^{3}\left(T_{3,5} \# D\right)$ and prove that it does not split in the cobordism group.

In this section and the next, and also in Appendix B, we develop properties of the Heegaard-Floer complex of specific torus knots and tensor products of certain of these complexes. More extensive computations appear in [Hancock et al. 2013].

10.1. $\left.\bar{d}\left(S_{15}^{3}\left(T_{3,5}\right), i\right)\right)$. We now determine the doubly filtered Heegaard-Floer complex $\operatorname{CFK}^{\infty}\left(S^{3}, T_{3,5}\right)$. This complex is by definition a doubly filtered, graded chain complex over $\mathbb{F}_{2}$. Thus a set of filtered generators can be illustrated on a grid with the coordinates representing the filtration levels and the grading marked. There is an action of $\mathbb{Z}$ on the complex, and if we let $U$ be the generator, this makes the complex a $\mathbb{F}_{2}\left[U, U^{-1}\right]$-module. The action of $U$ on the complex lowers filtration levels by 1 and gradings by 2 .

We now show that $\mathrm{CFK}^{\infty}\left(S^{3}, T_{3,5}\right)$ is as illustrated in Figure 2. In order to find this decomposition, we start by focusing on the central column (for which the top-most generator is at filtration level $j=4$ and is labeled with its grading 0 ). The vertical column, $i=0$, represents the subquotient complex $\widehat{\operatorname{CFK}}\left(S^{3}, T_{3,5}\right)$. We begin by explaining why it appears as it does in the illustration. According to [Ozsváth and Szabó 2005, Theorem 1.2], since for torus knots there is an integer surgery that yields a lens space, $\widehat{\operatorname{HFK}}\left(S^{3}, T_{3,5}, j\right)$, the quotient of the $j$-filtration level by the $(j-1)$-filtration level is completely determined by the Alexander polynomial,

$$
\Delta_{T_{3,5}}(t)=1-\left(t^{-1}+t\right)+\left(t^{-3}+t^{3}\right)-\left(t^{-4}+t^{4}\right) .
$$

This explains the location of the generators of $\widehat{\mathrm{CFK}}\left(S^{3}, T_{3,5}\right)$. Similarly the same work determines the grading of the generators. The fact the complex $\widehat{\mathrm{CFK}}\left(S^{3}, T_{3,5}\right)$ is a filtration of the complex $\widehat{C F}\left(S^{3}\right)$, which has homology $\mathbb{F}_{2}$ with its generator at grading level 0 , forces the vertical arrows, presenting the boundary maps, to be as illustrated. To build the $\mathrm{CFK}^{\infty}$ diagram from the $\widehat{\mathrm{CFK}}$ diagram, we first apply the action of $U$ to fill in the generators as well as the all the vertical arrows. We next note that the homology groups $\widehat{\operatorname{HFK}}\left(T_{3,5}, i\right)$ can be computed using the horizontal slice $j=0$ instead of the vertical slice, and this forces the existence of the horizontal arrows as drawn. With this much of the diagram drawn and the action of $U$ lowering grading by 2 , the gradings of all the elements in the diagram are determined. Finally we note that the fact that the boundary map lowers gradings by 1 rules out the possibility of any other arrows. 


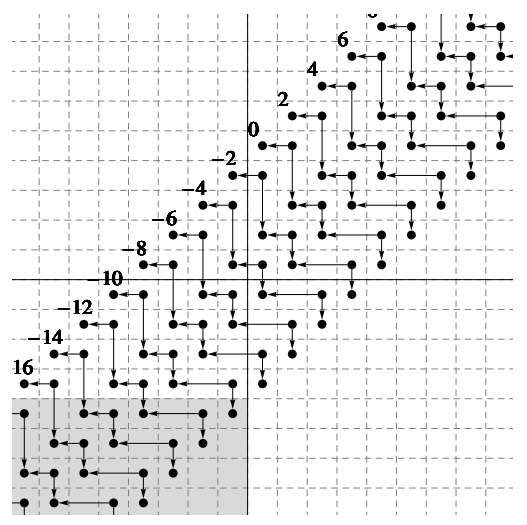

Figure 2. The case $s=-4$ with the quotienting subgroup shaded.

According to [Ozsváth and Szabó 2004], the complex $\mathrm{CFK}^{+}\left(S_{15}^{3}\left(T_{3,5}\right), s\right)$, for $-7 \leq s \leq 7$, is given by the quotient

$$
\mathrm{CFK}^{\infty}\left(S^{3}, T_{3,5}\right) / \mathrm{CFK}^{\infty}\left(S^{3}, T_{3,5}\right)_{i<0, j<s}[-\eta],
$$

where $\eta$ is a grading shift:

$$
\eta=\frac{-(2 s-15)^{2}+15}{60} .
$$

Figure 2 illustrates the case $s=-4$ with the quotienting subgroup shaded in the diagram. By definition, the $d$-invariant is the minimal grading among all classes in the group $\mathrm{HFK}^{+}\left(S_{15}^{3}\left(T_{3,5}\right), s\right)$, which are in the image of $U^{n}$ for all $n$. From the diagram, without shifting the gradings, we see this minimum for $\mathrm{HFK}^{+}\left(S_{15}^{3}\left(T_{3,5}\right),-4\right)$ is -8 : one generator of grading level -10 has been killed, and all such generators are homologous. The values for all $\operatorname{Spin}^{c}$ structures, $s=-7,-6, \ldots, 6,7$ are given in order as

$$
\{-14,-12,-10,-8,-8,-6,-4,-4,-2,-2,-2,0,0,0,0\} .
$$

After the grading shift, the values are all of the form $a_{i} / 30$, where, in order, the $a_{i}$ are

$$
\{-7,-3,5,17,-27,-7,17,-15,17,-7,-27,17,5,-3,-7\} \text {. }
$$

Finally to compute $\bar{d}$, we subtract $-15 / 30$ (the value for the spin structure) from each entry and find that the values of $\bar{d}$ are given by $b_{i} / 30$ for the following values of $b_{i}$ in order:

$$
\{8,12,20,32,-12,8,32,0,32,8,-12,32,20,12,8\} .
$$

We have listed these values in Table 3 , in which we write each value of $s$ as $5 a+3 b$ $\bmod 15$ for $-1 \leq a \leq 1$ and $-2 \leq b \leq 2$. 


\begin{tabular}{rrrrrrr}
$a$ & $b=-2$ & -1 & 0 & 1 & 2 \\
\hline 1 & 32 & 8 & $\mathbf{2 0}$ & 8 & 32 \\
0 & $\mathbf{1 2}$ & $\mathbf{- 1 2}$ & $\mathbf{0}$ & $\mathbf{- 1 2}$ & $\mathbf{1 2}$ \\
-1 & 32 & 8 & $\mathbf{2 0}$ & 8 & 32 \\
\hline
\end{tabular}

Table 3. $30 \bar{d}\left(S_{15}^{3}\left(T_{3,5}\right), 5 a+3 b\right)$.

Since $S_{15}^{3}\left(T_{3,5}\right)$ is the connected sum of lens spaces, Theorem 9 predicts a pattern in the chart: each element should be the sum of the entries of its projection on the main axes. This is the case. Notice for instance that the top-right entry 32 in position $(a, b)=(1,2) \in \mathbb{Z}_{3} \oplus \mathbb{Z}_{5}$ (which represents $1(5)+2(3)=11 \in \mathbb{Z}_{15}$ ) is the sum of the entries in positions $(2,0)$ and $(0,1), 12$ and 20 , respectively.

10.2. $\bar{d}\left(S_{15}^{3}\left(T_{3,5} \# D\right), \mathfrak{s}\right)$. In order to compute the $\bar{d}$-invariants that are associated to surgery on the connected sum, we first must compute $\mathrm{CFK}^{\infty}$ for the connected sum of knots. The complex $\operatorname{CFK}^{\infty}\left(T_{2,3}\right)$ is illustrated in Figure 3, left, and it follows from [Hedden 2007] that, modulo acyclic subcomplexes, the chain complex for $D$ is the same. (The results of the same article are focused on the hat complex $\widehat{\mathrm{CFK}(K)}$ but extend to the full $\mathrm{CFK}^{\infty}$ complex. For more details, see [Hedden et al. 2014].)

At this point we need to analyze the tensor product

$$
C=\mathrm{CFK}^{\infty}\left(T_{3,5}\right) \otimes_{\mathbb{F}\left[U, U^{-1}\right]} \mathrm{CFK}^{\infty}\left(T_{2,3}\right) .
$$

This complex is fairly complicated, containing 21 generators, but it is easily seen that it contains a subcomplex $C^{\prime}$ as illustrated in Figure 3, right. This subcomplex
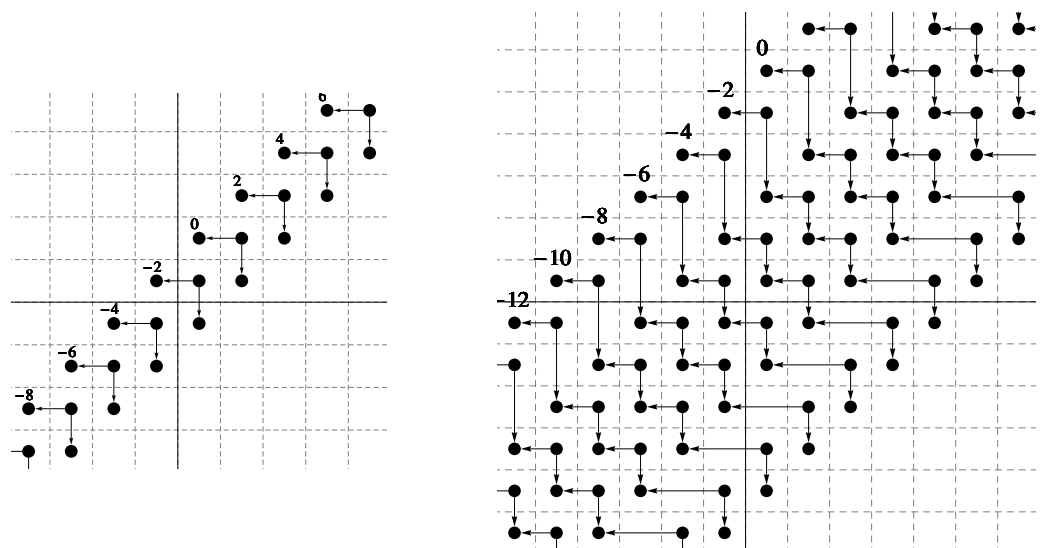

Figure 3. Left: the complex $\mathrm{CFK}^{\infty}\left(T_{2,3}\right)$. Right: The subcomplex $C^{\prime}$ of $\mathrm{CFK}^{\infty}\left(T_{3,5}\right) \otimes_{\mathbb{F}\left[U, U^{-1}\right]} \mathrm{CFK}^{\infty}\left(T_{2,3}\right)$. 


\begin{tabular}{rrrrrrr}
$a$ & $b=-2$ & -1 & 0 & 1 & 2 \\
\hline 1 & $\underline{-28}$ & 8 & $\mathbf{2 0}$ & 8 & $\underline{-28}$ \\
0 & $\mathbf{1 2}$ & $\mathbf{- 1 2}$ & $\mathbf{0}$ & $\mathbf{- 1 2}$ & $\mathbf{1 2}$ \\
-1 & $\underline{-28}$ & 8 & $\mathbf{2 0}$ & 8 & $\underline{-28}$ \\
\hline
\end{tabular}

Table 4. $30 \bar{d}\left(S_{15}^{3}\left(T_{3,5} \# D\right), 5 a+3 b\right)$.

carries the homology of the overall complex but does not contain all generators of a given grading. However, by examining the full complex with 21 generators, we have the following observation.

The complex $C_{i<m, j<n}$ contains a generator of grading 0 if and only if $C_{i<m, j<n}^{\prime}$ contains a generator of grading 0 . In particular, $d$-invariants for $C$ can be computed using $C^{\prime}$.

Using this diagram to compute the minimal gradings of classes in

$$
\operatorname{CFK}^{\infty}\left(T_{3,5} \# D\right) / \operatorname{CFK}^{\infty}\left(T_{3,5} \# D\right)_{i<0, j<s}
$$

for $-7 \leq s \leq 7$, we get the following:

$$
\{-14,-12,-10,-10,-8,-6,-6,-4,-4,-2,-2,-2,0,0,0\} \text {. }
$$

After shifting gradings by $-\eta$, the values are of the form $a_{i} / 30$, where the $a_{i}$ are, in order,

$$
\{-7,-3,5,-43,-27,-7,-43,-15,-43,-7,-27,-43,5,-3,-7\} .
$$

To compute $\bar{d}$, we add $15 / 30$ to each term, yielding the values $b_{i} / 30$, where the $b_{i}$ are

$$
\{8,12,20,-28,-12,8,-28,0,-28,8,-12,-28,20,12,8\} .
$$

We can arrange these in a chart as in Table 4.

Notice that the entries on the axes are unchanged, but the underlined entries are no longer the sum of the values of the projections; that is, $-28 \neq 12+20$. Thus, according to Theorem 9 , this manifold is not $\mathbb{Q}$-homology cobordant to any manifold of the form $M_{3} \# M_{5} \# M_{q}$.

10.3. Second example. As a second example we consider the case of $S_{35}^{3}\left(T_{5,7}\right)$ and $S_{35}^{3}\left(T_{5,7} \# D\right)$ and illustrate the analogous charts as above (this time multiplied by 70 to clear denominators). The first chart, Table 5 , necessarily demonstrates additivity; the second, in Table 6, upon examination does not. This becomes more apparent by considering the third chart, in Table 7, formed as the difference of the first two, but not multiplied by 70 . The underlined entries illustrate the failure of additivity. Considering this difference is a simplifying approach of the general proof in the next section. 


\begin{tabular}{r|rrrrrrr}
$a$ & $b=-3$ & -2 & -1 & 0 & 1 & 2 & 3 \\
\hline 2 & -68 & -108 & -48 & $\mathbf{- 2 8}$ & -48 & -108 & -68 \\
1 & -12 & -52 & 8 & $\mathbf{2 8}$ & 8 & -52 & -12 \\
0 & $\mathbf{- 4 0}$ & $\mathbf{- 8 0}$ & $\mathbf{- 2 0}$ & $\mathbf{0}$ & $\mathbf{- 2 0}$ & $\mathbf{- 8 0}$ & $\mathbf{- 4 0}$ \\
-1 & -12 & -52 & 8 & $\mathbf{2 8}$ & 8 & -52 & -12 \\
-2 & -68 & -108 & -48 & $\mathbf{- 2 8}$ & -48 & -108 & -68 \\
\hline
\end{tabular}

Table 5. $70 \bar{d}\left(S_{35}^{3}\left(T_{5,7}\right), 7 a+5 b\right)$.

\begin{tabular}{r|rrrrrrr}
$a$ & $b=-3$ & -2 & -1 & 0 & 1 & 2 & 3 \\
\hline 2 & $\underline{72}$ & $\underline{32}$ & 92 & $\mathbf{1 1 2}$ & 92 & $\underline{32}$ & $\underline{72}$ \\
1 & 128 & 88 & 8 & $\mathbf{2 8}$ & 8 & 88 & 128 \\
0 & $\mathbf{1 0 0}$ & $\mathbf{6 0}$ & $\mathbf{- 2 0}$ & $\mathbf{0}$ & $\mathbf{- 2 0}$ & $\mathbf{6 0}$ & $\mathbf{1 0 0}$ \\
-1 & 128 & 88 & 8 & $\mathbf{2 8}$ & 8 & 88 & 128 \\
-2 & $\underline{72}$ & $\underline{32}$ & 92 & $\mathbf{1 1 2}$ & 92 & $\underline{32}$ & $\underline{72}$ \\
\hline
\end{tabular}

Table 6. $70 \bar{d}\left(S_{35}^{3}\left(T_{5,7} \# D\right), 7 a+5 b\right)$.

\begin{tabular}{|c|c|c|c|c|c|c|c|}
\hline$a$ & & -2 & -1 & 0 & 1 & 2 & 3 \\
\hline 2 & $\underline{2}$ & $\underline{2}$ & 2 & 2 & 2 & $\underline{2}$ & $\underline{2}$ \\
\hline 1 & 2 & 2 & 0 & 0 & ( & 2 & 2 \\
\hline 0 & 2 & 2 & 0 & 0 & ( & 2 & $z$ \\
\hline-1 & 2 & 2 & 0 & 0 & ( & 2 & 2 \\
\hline-2 & $\underline{2}$ & $\underline{2}$ & 2 & 2 & 2 & $\underline{2}$ & $\underline{2}$ \\
\hline
\end{tabular}

Table 7. $\bar{d}\left(S_{35}^{3}\left(T_{5,7} \# D\right), 7 a+5 b\right)-\bar{d}\left(S_{35}^{3}\left(T_{5,7}\right), 7 a+5 b\right)$.

\section{Topologically split examples, general case}

We now wish to generalize the examples of the previous section. To do so, we begin by choosing an infinite set of integers $\left\{p_{i}\right\}$ with the following properties: (1) all the $p_{i}$ are odd; (2) the elements of the full set $\left\{p_{i}\right\} \cup\left\{p_{i}+2\right\}$ are pairwise relatively prime; and (3) each $p_{i}$ and $p_{i}+2$ is square-free. The existence of such a set is demonstrated in Appendix A, and throughout this section we assume all $p$ are selected from this set. In the previous example we needed to track grading shifts. It will simplify our discussion if we avoid dealing the grading shifts as follows. Define $\tilde{d}\left(S_{n}^{3}(K), s\right)=d\left(S_{n}^{3}(K), s\right)+\eta$. That is, $\tilde{d}$ is computed as is the $d$-invariant, except without the grading shift, the induced grading on

$$
\mathrm{CFK}^{+}\left(S_{N}^{3}(K), s\right)=\mathrm{CFK}^{\infty}\left(S^{3}, K\right) / \mathrm{CFK}^{\infty}\left(S^{3}, K\right)_{\{i<0, j<s\}} .
$$


Since $p$ is odd, we can write $p=2 n+1$ and let $q=p+2=2 n+3$. Our manifolds of interest are $S_{p q}^{3}\left(T_{p, q}\right)$ and $S_{p q}^{3}\left(T_{p, q} \# D\right)$. We collect here the results of a few elementary calculations.

\section{Theorem 16.}

(1) The surgery coefficient is $p q=4 n^{2}+8 n+3$.

(2) The three-genus satisfies

$$
g\left(T_{p, q}\right)=2 n(n+1)=2 n^{2}+2 n \quad \text { and } \quad g\left(T_{p, q} \# D\right)=2 n^{2}+2 n+1 .
$$

(3) $\operatorname{Spin}^{c}$ structures are parameterized by s, with

$$
-\left(2 n^{2}+4 n+1\right) \leq s \leq\left(2 n^{2}+4 n+1\right) .
$$

(4) Generators of $\widehat{\mathrm{CFK}}\left(T_{p, q}\right)$ have filtration level $j$, where

$$
-2 n(n+1) \leq j \leq 2 n(n+1) .
$$

The main result of this section is the following.

Theorem 17. $\bar{d}\left(S_{p q}^{3}\left(T_{p, q} \# D\right), s\right)$ does not satisfy additivity as given in Theorem 9. Proof. The space $S_{p q}^{3}\left(T_{p, q}\right)$ satisfies the additive property as in Theorem 9. Suppose that $S_{p q}^{3}\left(T_{p, q} \# D\right)$ also satisfies the additivity property. Then the difference $\bar{d}\left(S_{p q}^{3}\left(T_{p, q}\right),(a, b)\right)-\bar{d}\left(S_{p q}^{3}\left(T_{p, q} \# D\right),(a, b)\right)$ also satisfies the additivity property. We denote this difference by $\bar{d}^{\prime}(a, b)$ or $\bar{d}^{\prime}(a q+b p)$. Note that it is unnecessary to add the grading shift $\eta$ to the amount we get from the diagram when computing either of the values $\bar{d}\left(S_{p q}^{3}\left(T_{p, q}\right),(a, b)\right)$ or $\bar{d}\left(S_{p q}^{3}\left(T_{p, q} \# D\right),(a, b)\right)$ since they have the same grading shift. Namely

$$
\begin{gathered}
\bar{d}^{\prime}(a, b)=\tilde{d}\left(S_{p q}^{3}(T(p, q)),(a, b)\right)-\tilde{d}\left(S_{p q}^{3}\left(T_{p, q} \# D\right),(a, b)\right) \\
-\tilde{d}\left(S_{p q}^{3}\left(T_{p, q}\right), 0\right)+\tilde{d}\left(S_{p q}^{3}\left(T_{p, q} \# D\right), 0\right) .
\end{gathered}
$$

From our choice of $p$ and $q$, we have $(n+1) p+(-n) q=1$. Thus the additivity property implies the equality

$$
\bar{d}^{\prime}(1)=\bar{d}^{\prime}((n+1) p)+\bar{d}^{\prime}(-n q),
$$

or, equivalently,

$$
\begin{aligned}
(11-1) \tilde{d}\left(S_{p q}^{3}\left(T_{p, q}\right), 1\right)-\tilde{d} & \left(S_{p q}^{3}\left(T_{p, q} \# D\right), 1\right) \\
=\tilde{d}( & \left.S_{p q}^{3}\left(T_{p, q}\right),(n+1) p\right)-\tilde{d}\left(S_{p q}^{3}\left(T_{p, q} \# D\right),(n+1) p\right) \\
& +\tilde{d}\left(S_{p q}^{3}\left(T_{p, q}\right),-n q\right)-\tilde{d}\left(S_{p q}^{3}\left(T_{p, q} \# D\right),-n q\right) \\
& -\tilde{d}\left(S_{p q}^{3}\left(T_{p, q}\right), 0\right)+\tilde{d}\left(S_{p q}^{3}\left(T_{p, q} \# D\right), 0\right) .
\end{aligned}
$$


Since $(n+1) p=2 n^{2}+3 n+1$ lies between the genus of $T(p, q)$ (and of $T_{p, q} \# D$ ) and the upper bound on the parameters for the $\operatorname{Spin}^{c}$ structures,

$$
2 n^{2}+2 n+1<2 n^{2}+3 n+1<2 n^{2}+4 n+1,
$$

the values of the $\tilde{d}$-invariants are easily seen to be 0 . On the other hand, the number $-n q$ is greater than the lower bound on the parameters for the $\operatorname{Spin}^{c}$ structures and less than the negative of the genus,

$$
-\left(2 n^{2}+4 n+1\right)<-\left(2 n^{2}+3 n\right)<-\left(2 n^{2}+2 n+1\right),
$$

and thus one sees that the $\tilde{d}$-invariants associated to $-n q$ take the same value $-2 s=2\left(2 n^{2}+3 n\right)$ for both $T_{p, q}$ and $T_{p, q} \# D$. Thus, in contradicting additivity, it remains to show that the equality

$\tilde{d}\left(S_{p q}^{3}\left(T_{p, q}\right), 1\right)-\tilde{d}\left(S_{p q}^{3}\left(T_{p, q} \# D\right), 1\right)=-\tilde{d}\left(S_{p q}^{3}\left(T_{p, q}\right), 0\right)+\tilde{d}\left(S_{p q}^{3}\left(T_{p, q} \# D\right), 0\right)$

does not hold.

Now we will compute $\tilde{d}$ of both spaces for $\operatorname{Spin}^{c}$ structures 0 and 1 . Observe that within width 1 from the diagonal $j=i$, the complex $\operatorname{CFK}^{\infty}\left(S^{3}, T_{p, q}\right)$ looks like $\mathrm{CFK}^{\infty}\left(S^{3}, T_{2,3}\right)$ if $n$ is odd or $\operatorname{CFK}^{\infty}\left(S^{3}, T_{2,5}\right)$ if $n$ is even. This depends on the fact that near the origin the complex $\operatorname{CFK}^{\infty}\left(S^{3}, T_{p, q}\right)$ looks like that of the $(2, k)$-torus knots. In Appendix B we prove that the Alexander polynomial of $T_{p, p+2}$ is of the form $1+\sum_{i>0} a_{i}\left(t^{-i}+t^{i}\right)$, where $a_{i}= \pm 1$ for $i \leq(p-1) / 2$. As in the example of the previous section, this determines the "zig-zag" feature of the $\mathrm{CFK}^{\infty}$ complex near the origin. Tensoring with the trefoil complex does not alter this pattern.

The generators of the same grading $2 l$ of $[x,-1,0]$ if $n$ is odd (or $[x, 0,0]$ if $n$ is even) lies above the antidiagonal $i+j=-1$ (or $i+j=0$ ). So, in order to compute $\tilde{d}\left(S_{p q}^{3}(T(p, q)), s\right)$ for $s=0,1$, we may assume in the computations that the complex we are considering is one of

$$
\begin{cases}\operatorname{CFK}^{\infty}\left(S^{3}, T_{2,3}\right) & \text { if } n \text { is odd, } \\ \operatorname{CFK}^{\infty}\left(S^{3}, T_{2,5}\right) & \text { if } n \text { is even. }\end{cases}
$$

It is now easy to compute

$$
\tilde{d}\left(S_{p q}^{3}\left(T_{p, q}\right), s\right)=\begin{array}{c|cc}
s & n \text { odd } & n \text { even } \\
\hline 1 & 2 l+2 & 2 l \\
0 & 2 l & 2 l
\end{array}
$$

Near the diagonal $j=i$, the complex $\mathrm{CFK}^{\infty}\left(S^{3}, T_{p, q} \# D\right)$ looks like

$$
\begin{cases}\operatorname{CFK}^{\infty}\left(S^{3}, T_{2,5}\right) & \text { if } n \text { is odd, } \\ \operatorname{CFK}^{\infty}\left(S^{3}, T_{2,3}\right)[-2] & \text { if } n \text { is even. }\end{cases}
$$


The grading of $[x,-1,0]$ is $2 l-2$ if $n$ is even and the grading of $[x, 0,0]$ is $2 l$ if $n$ is odd. Thus we have

$$
\tilde{d}\left(S_{p q}^{3}\left(T_{p, q} \# D\right), s\right)=\begin{array}{c|cc}
s & n \text { odd } & n \text { even } \\
\hline 1 & 2 l & 2 l \\
0 & 2 l & 2 l-2
\end{array}
$$

We see that

$$
\tilde{d}\left(S_{p q}^{3}\left(T_{p, q}\right), s\right)-\tilde{d}\left(S_{p q}^{3}\left(T_{p, q} \# D\right), s\right)=\begin{array}{c|cc}
s & n \text { odd } & n \text { even } \\
\hline 1 & 2 & 0 \\
0 & 0 & 2
\end{array}
$$

This shows that (11-1) cannot be satisfied. We conclude that the space $S_{p q}^{3}\left(T_{p, q} \# D\right)$ does not satisfy the additive property of Theorem 9 .

11.1. The image of $\mathscr{Y}$ in $\Theta_{\mathbb{Q}}^{3} / \Phi\left(\bigoplus \Theta_{p}\right)$ is infinite. This is a consequence of the following result.

Theorem 18. The spaces $N_{p, q}=S_{p q}^{3}\left(T_{p, q} \# D\right) \#-S_{p q}^{3}\left(T_{p, q}\right) \in \mathscr{K}$ are distinct in the quotient $\Theta_{\mathbb{Q}}^{3} / \Phi\left(\bigoplus \Theta_{p}\right)$.

Proof. Observe that $S_{p q}^{3}\left(T_{p, q} \# D\right) \#-S_{p q}^{3}\left(T_{p, q}\right) \in \mathscr{K}$ since the knots are topologically concordant. We next observe that these manifolds have the property that no linear combination with all coefficients \pm 1 is trivial in the quotient. Suppose that some such linear combination was trivial. Then focusing on any particular pair $(p, q)$, we would have that $S_{p q}^{3}\left(T_{p, q} \# D\right) \# M_{p} \# M_{q} \# M_{m}=\partial X$ for a rational homology ball $X$, where the order of $M_{p}$ is a product of prime factors of $p$, the order of $M_{q}$ is a product of prime factors of $q$, and the order of $M_{m}$ is relatively prime to $p q$. (This uses the fact that $S_{p q}^{3}\left(T_{p, q}\right)$ does split as a connected sum.)

The existence of this connected sum decomposition implies the additivity for $d$-invariants of $S_{p q}^{3}\left(T_{p, q} \# D\right)$ in a way that contradicts Theorem 17.

\section{Knot concordance}

We denote by $\mathscr{C}$ the classical smooth knot concordance group. Levine [1969] defined the algebraic concordance group $\mathscr{G}$ and the rational algebraic concordance group, $\mathscr{G}^{\mathbb{Q}}$. He also defined a surjective homomorphism $\mathscr{b} \rightarrow \mathscr{G}$, proved that the natural map $\mathscr{G} \rightarrow \mathscr{G}^{\mathbb{Q}}$ is injective, and proved that $\mathscr{G}^{\mathbb{Q}}$ is isomorphic to an infinite direct sum of groups isomorphic to $\mathbb{Z}, \mathbb{Z}_{2}$ and $\mathbb{Z}_{4}$. He also proved that the image of $\varphi$ in $\mathscr{G}^{\mathbb{Q}}$ is isomorphic to a similar infinite direct sum. In the same article it is observed that $\mathscr{G}^{\mathbb{Q}}$ has a natural decomposition as a direct sum $\oplus_{p}^{\mathscr{Q}^{\mathbb{Q}}(t)}$, where the $p(t)$ are symmetric irreducible rational polynomials. We will not present the details here, but note that if the Alexander polynomial of $K, \Delta_{K}(t)$, is irreducible, 
then the image of $K$ in $\mathscr{G}^{\mathbb{Q}}$ is in the $\mathscr{G}_{\Delta(t)}^{\mathbb{Q}}$ summand. Stoltzfus [1977] observed that the algebraic concordance group $\mathscr{G}$ does not have a similar splitting. Thus there is not an immediate analog in concordance for the decompositions we have been studying for homology cobordism. However he did prove that in some cases such a splitting exists. The following, from Corollary 6.5 of the same work, is stated in terms of knot concordance, but, given the isomorphism of higher-dimensional concordance and $\mathscr{G}^{\mathbb{Z}}$, the same splitting theorem holds in the algebraic concordance group.

Theorem 19. If $K$ is an $n$-dimensional knot for $n>1$ and $\Delta_{K}(t)$ factors as $p(t) q(t)$ with $p(t)$ and $q(t)$ symmetric and the resultant $\operatorname{Res}(p(t), q(t))=1$, then $K$ is concordant to a connected sum $K_{1} \# K_{2}$, with $\Delta_{K_{1}}(t)=p(t)$ and $\Delta_{K_{2}}(t)=q(t)$.

Here we observe that this result does not hold in dimension 3.

Example. Consider the ten-crossing knot $K=10_{5}$. It has Alexander polynomial

$$
\Delta=\left(1-t+t^{2}\right)\left(1-2 t+2 t^{2}-t^{3}+2 t^{4}-2 t^{5}+t^{6}\right) .
$$

These two factors are irreducible and have resultant 1 .

Theorem 20. The knot $10_{5}$ is not concordant to any connected sum $K_{1} \# K_{2}$, where $\Delta_{K_{1}}=1-t+t^{2}$ and $\Delta_{K_{2}}=1-2 t+2 t^{2}-t^{3}+2 t^{4}-2 t^{5}+t^{6}$.

Proof. The 2-fold branched cover of $K$ is the lens space $L(33,13)$. If the desired concordance existed, then $L(33,13)$ would split in rational cobordism as a connected sum $M_{3} \# M_{11}$, with $H_{1}\left(M_{3}\right)=\mathbb{Z}_{3}$ and $H_{1}\left(M_{11}\right)=\mathbb{Z}_{11}$. In order to compute the relevant $d$-invariants, one first identifies $\mathfrak{s}_{6}$ as the spin structure $\mathfrak{s}_{*}$ by computing that the value of $d\left(L(33,13), \mathfrak{s}_{6}\right)=33$, a value that is not attained by any other $\operatorname{Spin}^{c}$ structure. The values of the $d$-invariants, $d\left(L(33,13),(a, b) \cdot \mathfrak{s}_{*}\right)-d\left(L(33,13), \mathfrak{s}_{*}\right)$ for $(a, b) \in \mathbb{Z}_{3} \oplus \mathbb{Z}_{11}$ are given in the chart in Table 8 (multiplied by 33 to clear denominators).

The next chart, in Table 9, presents the values

$$
\begin{aligned}
\delta(L(33,13),(a, b))=d & (L(33,13),(a, b))-d(L(33,13),(a, 0)) \\
& -a d(L(33,13),(0, b))+d(L(33,13),(0,0)) .
\end{aligned}
$$

\begin{tabular}{r|rrrrrr}
$a$ & $b=0$ & 1 & 2 & 3 & 4 & 5 \\
\hline 1 & $\mathbf{2 2}$ & 10 & 40 & 46 & 28 & 52 \\
0 & $\mathbf{0}$ & $\mathbf{5 4}$ & $\mathbf{1 8}$ & $\mathbf{2 4}$ & $\mathbf{6}$ & $\mathbf{3 0}$ \\
-1 & $\mathbf{2 2}$ & 10 & 40 & -20 & 28 & -14 \\
\hline
\end{tabular}

Table 8. $33 d(L(33,13), 11 a+3 b)$. 


\begin{tabular}{|c|c|c|c|c|c|c|}
\hline$a$ & & 1 & 2 & 3 & 4 & 5 \\
\hline 1 & 0 & 2 & 0 & 0 & 0 & 0 \\
\hline 0 & 0 & 0 & 0 & 0 & 0 & 0 \\
\hline-1 & 0 & 2 & 0 & 2 & 0 & 2 \\
\hline
\end{tabular}

Table 9. $\delta(L(33,13),(a, b))$.

The presence of the nonzero entries implies the nonsplittability of the manifold, as desired.

Note. In [Livingston 2002], the second author constructed similar but much more complicated examples in the topological category.

\section{Topologically trivial bordism}

In [Hedden et al. 2012] the quotient $\Theta_{\mathbb{Q}, \text { spin }}^{T} / \Theta_{\mathbb{Q}, \text { spin }}^{I}$ was studied. Here the cobordism group has been restricted to spin 3-manifolds and spin bordisms which have the rational homology of $S^{3}$. The notation $\Theta_{\mathbb{Q} \text {,spin }}^{T}$ denotes the subgroup generated by representatives which bound topological homology balls and $\Theta_{\mathbb{Q} \text {,spin }}^{I}$ is generated by those that are cobordant to $\mathbb{Z}$-homology spheres. (Note that we have changed the notation from that of the same reference to be consistent with the results of the current paper.) There is also a similar result in the same work replacing ( $\mathbb{Q}$, spin), with $\mathbb{Z}_{2}$. Recall that every orientable 3-manifold has trivial tangent bundle, so is spin, and that every $\mathbb{Z}_{2}$ homology 3 -sphere has a unique spin structure.

Here we observe that Theorem 6 permits us to eliminate the need to constrain the cobordism group to being spin or to use $\mathbb{Z}_{2}$ coefficients. Let $\Theta_{\mathbb{Q}}^{T}$ denote the subgroup of $\Theta_{\mathbb{Q}}^{3}$ generated by rational homology spheres that are trivial in the topological rational cobordism group, that is, the kernel of $\mathscr{K}$.

Theorem 21. The quotient group $\Theta_{\mathbb{Q}}^{T} / \Theta_{\mathbb{Z}}^{3}$ is infinitely generated.

We outline how the argument in [Hedden et al. 2012] can be generalized.

In this work there is a family of rational homology spheres, $M_{p^{2}}$, constructed for an infinite set of primes $p$. These are constructed so that they bound topological balls. The proof of the theorem consists of showing that no linear combination $N=\#_{i} a_{i} M_{p_{i}^{2}} \# M_{0}$ bounds a spin rational homology ball (or $\mathbb{Z}_{2}$ homology ball) $W$, where $M_{0}$ is a $\mathbb{Z}$-homology sphere. The existence of a unique spin structure was used to identify $\operatorname{Spin}^{c}$ of the relevant manifolds with the second homology.

If all $p$ are odd, then there is a unique $\operatorname{Spin}^{c}$ structure on $N$, and according to Theorem 6, it is the restriction of a $\operatorname{Spin}^{c}$ structure on $W$. Given this, Proposition 2.1 of the same reference, which required that $W$ be spin, continues to apply to identify the $\operatorname{Spin}^{c}$ structures on $N$ which extend to $W$ with a metabolizer of the linking form on $H_{1}(N)$. That identification is what is used to obstruct the existence 
of $W$ via $d$-invariants, as described in Theorem 3.2 of the same work. Thus the remainder of the proof goes through as in that paper.

\section{Appendix A. Finding the $p_{i}$}

The proof of Theorem 17 requires a sequence of odd pairs $\left\{p_{i}, p_{i}+2\right\}$ such that the elements of the full set $\left\{p_{i}\right\} \cup\left\{p_{i}+2\right\}$ are pairwise relatively prime and square-free. Since $p_{i}$ and $p_{i}+2$ are relatively prime, we need to choose the $p_{i}$ so that all elements of $\left\{p_{i}\left(p_{i}+2\right)\right\}$ are pairwise relatively prime and each element is square-free. If we let $p_{i}=n_{i}-1$, then $p_{i}\left(p_{i}+2\right)=n_{i}^{2}-1$, and so we are seeking an infinite sequence of positive integers $\left\{n_{i}\right\}$ such that:

(1) $n_{i}$ is even for all $i$.

(2) All elements of $\left\{n_{i}^{2}-1\right\}$ are relatively prime.

(3) Each $n_{i}^{2}-1$ is square-free.

In Section 9 we need a sequence of integers $n_{i}$ such that $n_{i}=0 \bmod 5$ with the property that the integers $20 n_{i}^{2}+8 n_{i}+1$ are relatively prime and square-free. Here is a theorem that covers both cases.

Theorem 22. Let $f(x) \in \mathbb{Z}[t]$ be an quadratic polynomial with constant term 1 that is not the square of a linear polynomial. Let $\alpha$ be a fixed integer and $s_{n}=\alpha n$ be an arithmetic sequence. There exists an infinite set of $s_{i}$ such that values of $f\left(s_{i}\right)$ are pairwise relatively prime and square-free.

Proof. It is known that if $g(n)$ is a quadratic polynomial that is not a square of a linear polynomial and which has the property that its coefficients have greatest common divisor one, then $g(n)$ is square-free for an infinite set of $n$ (see, for example, [Erdôs 1953]). We wish to construct the sequence of $s_{i}$ inductively. To find $s_{1}$, let $f_{1}(n)=f(\alpha n)$, which is irreducible with constant term one. Choose $n_{1}$ so that $f_{1}\left(n_{1}\right)$ is square-free. Let $s_{1}=\alpha n_{1}$. Assume that $s_{i}$ has been defined for $i<k$. We find $s_{k}$ with the desired properties as follows. Let $P=\prod_{i=1}^{k-1} f\left(s_{i}\right)$. Consider the function $f_{k}(n)=f(\alpha P n)$. Again this polynomial is irreducible with constant term one so there exists an $n_{k}$ for which $f_{k}\left(n_{k}\right)$ is square-free. Since $f_{k}\left(n_{k}\right)=f\left(\alpha P n_{k}\right)$, we let $s_{k}=\alpha P n_{k}$. Notice that $f(\alpha P n)=1 \bmod p$ for each prime divisor $p$ of $P$, since evaluating $f$ at $\alpha P n$ gives a quadratic polynomial in $n$, with the quadratic term and linear term divisible by $P$ and the constant term one. It follows that $f\left(s_{k}\right)$ is relatively prime to all $f\left(s_{i}\right), i<k$.

\section{Appendix B. The Alexander polynomial of $T_{p, p+2}$}

Normalized to be symmetric, the Alexander polynomial of a knot can be written in the form $\Delta_{K}(t)=a_{0}+\sum_{i=1}^{n} a_{i}\left(t^{-i}+t^{i}\right)$, where $a_{0}+2 \sum a_{i}= \pm 1$. In Section 11 we use the following fact. 
Theorem 23. If $K=T_{p, p+2}$ with $p$ odd then

$$
\Delta_{T_{p, p+2}}(t)=a_{0}+\sum_{i=1}^{\left(p^{2}-1\right) / 2} a_{i}\left(t^{-i}+t^{i}\right),
$$

where $a_{i}= \pm 1$ for $i \leq(p-1) / 2$.

Note. With more care, all the coefficients or $\Delta_{T_{p, p+2}}(t)$ can be described in closed form.

Proof. As a polynomial (as opposed to the normalized Laurent polynomial) with nonzero constant term, the Alexander polynomial of $T_{p, q}$ is

$$
\left(1-t^{p q}\right)(1-t) /\left(1-t^{p}\right)\left(1-t^{q}\right) .
$$

Expanding each term of the denominator in a power series and noting that multiplying by the $t^{p q}$ term in the numerators does not affect terms of the product of degree less than $2 g=(p-1)(q-1)$, the degree of the Alexander polynomial, we can focus on the expression

$$
(1-t)\left(1+t^{p}+t^{2 p}+t^{3 p}+\cdots\right)\left(1+t^{q}+t^{2 q}+\cdots\right),
$$

which we write as the product

$$
(1-t) \sum_{i=0}^{\infty} b_{i} t^{i}
$$

Here $b_{i}$ is the number of solutions to $x p+y q=i$, with $x, y \geq 0$. In the case of interest, $q=p+2$ and the genus $g=\left(p^{2}-1\right) / 2$. We will now show that for $i$ in the range $g-A \leq i \leq g$, the values $b_{i}$ are alternately 0 and 1 , where $A$ is a constant to be determined. Thus, using the fact that the Alexander polynomial is symmetric, upon multiplying by $(1-t)$ we have the coefficients of the Alexander polynomial are all \pm 1 near $t^{g}$. To show that the coefficients $b_{i}$ alternate between 0 and 1 for $g-A \leq i \leq g$, we first observe that in a given range of $i$, all $b_{i} \geq 1$ for $i$ even. To see this, write $p=2 n+1$ and $q=2 n+3$; thus $g=2 n^{2}+2 n$. Consider the sum

$$
\frac{n+j}{2} p+\frac{n-j}{2} q=2 n^{2}+2 n-j,
$$

where $j$ is selected to have the same parity as $n$. (We require here that $j \leq n$; that is, we need $A \leq(p-1) / 2$.) To complete the argument, we next observe that the difference $\left|b_{i}-b_{j}\right| \leq 1$ if $|i-j| \leq 1$. Suppose otherwise. That is, suppose that there are distinct nonnegative solutions to the equations

$$
x p+y q=i
$$


and

$$
x^{\prime} p+y^{\prime} q=j,
$$

with $x, y, x^{\prime}, y^{\prime} \geq 0,|i-j| \leq 1$, and $i, j \leq g$. The conditions that $i \leq g$ and $y \geq 0$ imply that $x p \leq g=(p q-p-q-1) / 2$, which implies that $x<(q-1) / 2$. We first consider the case that $i \neq j$. After possibly reordering, the difference would give

$$
\left(x-x^{\prime}\right) p+\left(y-y^{\prime}\right) q=1 .
$$

One solution to this equation is

$$
\frac{q-1}{2} p-\frac{p-1}{2} q=1 .
$$

Every other solution is given by adding a multiple of $(-q, p)$ to the coefficient vector (note that $-q(p)+p(q)=0$ is a primitive solution since $p$ and $q$ are relatively prime). Thus the solutions with the smallest absolute values of the $x$-coordinate to the unital equation are the one above and

$$
-\frac{q+1}{2} p+\frac{p+1}{2} q=1 .
$$

That is, the smallest possible value for $\left(x-x^{\prime}\right)$ is $x-x^{\prime}=(q-1) / 2$. But, since $x$ and $x^{\prime}$ both are nonnegative and less than $(q-1) / 2$, this is impossible. As an example, if $p=21$ and $q=23$ (so $g=220$ ), we have the solutions

$$
11(21)-10(23)=1
$$

and

$$
-12(21)+11(23)=1,
$$

with $g=220$. We also have $x(21)+y(23) \leq 220$ which implies that $x \leq 220 / 21$, so $0 \leq x \leq 10$. Similarly for $x^{\prime}$, so it is not possible for $\left|x-x^{\prime}\right|=11$. Finally we consider the case $i=j$. Thus our coefficients would satisfy

$$
\left(x-x^{\prime}\right) p+\left(y-y^{\prime}\right) q=0 .
$$

This implies that $x-x^{\prime}$ is a multiple of $q$. But this would imply that they are equal since under our assumptions both are nonnegative and also $x p \leq p q-p-q+1 \leq p q$, so $x<q$ and $x^{\prime}<q$.

In summary, if we write the Alexander polynomial of the $T_{p, q}$ torus knot, with $q-p=2$ as \pm 1 as $a_{0}+\sum_{i=1}^{g} a_{i}\left(t^{i}+t^{-i}\right)$, then for $i \leq(p-1) / 2$, we have shown that $a_{i}=(-1)^{i}$. 


\section{Acknowledgements}

We are grateful for Matt Hedden's help in better understanding Heegaard-Floer homology. His results regarding the Heegaard-Floer theory of doubled knots are central here, and our specific examples are inspired by those that Matt pointed us toward in our collaborations with him. We also thank the referee for offering significant improvement in the exposition.

\section{References}

[Alexander et al. 1976] J. P. Alexander, G. C. Hamrick, and J. W. Vick, "Linking forms and maps of odd prime order", Trans. Amer. Math. Soc. 221:1 (1976), 169-185. MR 53 \#6600 Zbl 0357.57009

[Casson and Gordon 1986] A. J. Casson and C. M. Gordon, "Cobordism of classical knots", pp. 181-199 in À la recherche de la topologie perdue, edited by L. Guillou and A. Marin, Progr. Math. 62, Birkhäuser, Boston, 1986. MR 900252 Zbl 0597.57001

[Erdős 1953] P. Erdős, "Arithmetical properties of polynomials", J. London Math. Soc. 28 (1953), 416-425. MR 15,104f Zbl 0051.27703

[Freedman 1982] M. H. Freedman, "The topology of four-dimensional manifolds", J. Differential Geom. 17:3 (1982), 357-453. MR 84b:57006 Zbl 0528.57011

[Freedman and Quinn 1990] M. H. Freedman and F. Quinn, Topology of 4-manifolds, Princeton Mathematical Series 39, Princeton University Press, 1990. MR 94b:57021 Zbl 0705.57001

[Furuta 1990] M. Furuta, "Homology cobordism group of homology 3-spheres", Invent. Math. 100:2 (1990), 339-355. MR 91c:57039 Zbl 0716.55008

[Gompf and Stipsicz 1999] R. E. Gompf and A. I. Stipsicz, 4-manifolds and Kirby calculus, Graduate Studies in Mathematics 20, American Mathematical Society, Providence, RI, 1999. MR 2000h:57038 Zbl 0933.57020

[Hancock et al. 2013] S. Hancock, J. Hom, and M. Newman, "On the knot Floer filtration of the concordance group", J. Knot Theory Ramifications 22:14 (2013), Article ID \#1350084. MR 3190122 Zbl 06273023

[Hedden 2007] M. Hedden, "Knot Floer homology of Whitehead doubles", Geom. Topol. 11 (2007), 2277-2338. MR 2008m:57030 Zbl 1187.57015

[Hedden et al. 2012] M. Hedden, C. Livingston, and D. Ruberman, "Topologically slice knots with nontrivial Alexander polynomial”, Adv. Math. 231:2 (2012), 913-939. MR 2955197 Zbl 1254.57008

[Hedden et al. 2014] M. Hedden, S. Kim, and C. Livingston, "Topologically slice knots of smooth concordance order two", preprint, 2014. arXiv 1212.6628

[Kawauchi and Kojima 1980] A. Kawauchi and S. Kojima, "Algebraic classification of linking pairings on 3-manifolds", Math. Ann. 253:1 (1980), 29-42. MR 82b:57007 Zbl 0427.57001

[Levine 1969] J. Levine, "Invariants of knot cobordism", Invent. Math. 8 (1969), 98-110. MR 40 \#6563 Zbl 0179.52401

[Lisca 2007] P. Lisca, "Sums of lens spaces bounding rational balls", Algebr. Geom. Topol. 7 (2007), 2141-2164. MR 2008m:57018 Zbl 1185.57015

[Livingston 2002] C. Livingston, "Examples in concordance", preprint, 2002. arXiv math/0101035v2

[Livingston and Naik 1999] C. Livingston and S. Naik, "Obstructing four-torsion in the classical knot concordance group”, J. Differential Geom. 51:1 (1999), 1-12. MR 2000g:57009 Zbl 1025.57013 
[Milnor and Husemoller 1973] J. Milnor and D. Husemoller, Symmetric bilinear forms, Ergebnisse der Mathematik und ihrer Grenzgebiete 73, Springer, New York, 1973. MR 58 \#22129 Zbl 0292.10016

[Ozsváth and Szabó 2003] P. S. Ozsváth and Z. Szabó, "Absolutely graded Floer homologies and intersection forms for four-manifolds with boundary", Adv. Math. 173:2 (2003), 179-261. MR 2003m:57066 Zbl 1025.57016

[Ozsváth and Szabó 2004] P. S. Ozsváth and Z. Szabó, "Holomorphic disks and knot invariants", Adv. Math. 186:1 (2004), 58-116. MR 2005e:57044 Zbl 1062.57019

[Ozsváth and Szabó 2005] P. S. Ozsváth and Z. Szabó, “On knot Floer homology and lens space surgeries”, Topology 44:6 (2005), 1281-1300. MR 2006f:57034 Zbl 1077.57012

[Stoltzfus 1977] N. W. Stoltzfus, Unraveling the integral knot concordance group, Mem. Amer. Math. Soc. 192, American Mathematical Society, Providence, RI, 1977. MR 57 \#7616 Zbl 0366.57005

Received June 17, 2013. Revised March 21, 2014.

\author{
SE-Goo Kim \\ KYUNG HEE UNIVERSITY \\ SEOUL 130-701 \\ SOUTH KOREA \\ sgkim@khu.ac.kr \\ CHARLES LiVINGSTON \\ DEPARTMENT OF MATHEMATICS \\ INDIANA UNIVERSITY \\ RAWLES HALL \\ BLOOMINGTON, IN 47405-5701 \\ UNITED STATES \\ livingst@indiana.edu
}

Department of Mathematics and Research Institute for Basic SCIENCES 


\title{
PACIFIC JOURNAL OF MATHEMATICS
}

\author{
msp.org/pjm
}

Founded in 1951 by E. F. Beckenbach (1906-1982) and F. Wolf (1904-1989)

\section{EDITORS}

Don Blasius (Managing Editor)

Department of Mathematics

University of California

Los Angeles, CA 90095-1555

blasius@math.ucla.edu

\author{
Paul Balmer \\ Department of Mathematics \\ University of California \\ Los Angeles, CA 90095-1555 \\ balmer@math.ucla.edu \\ Robert Finn \\ Department of Mathematics \\ Stanford University \\ Stanford, CA 94305-2125 \\ finn@math.stanford.edu \\ Sorin Popa \\ Department of Mathematics \\ University of California \\ Los Angeles, CA 90095-1555 \\ popa@math.ucla.edu
}

\author{
Vyjayanthi Chari \\ Department of Mathematics \\ University of California \\ Riverside, CA 92521-0135 \\ chari@math.ucr.edu \\ Kefeng Liu \\ Department of Mathematics \\ University of California \\ Los Angeles, CA 90095-1555 \\ liu@math.ucla.edu \\ Jie Qing \\ Department of Mathematics \\ University of California \\ Santa Cruz, CA 95064 \\ qing@ cats.ucsc.edu
}

\section{PRODUCTION}

Silvio Levy, Scientific Editor, production@msp.org

\section{SUPPORTING INSTITUTIONS}

ACADEMIA SINICA, TAIPEI

CALIFORNIA INST. OF TECHNOLOGY

INST. DE MATEMÁTICA PURA E APLICADA

KEIO UNIVERSITY

MATH. SCIENCES RESEARCH INSTITUTE

NEW MEXICO STATE UNIV.

OREGON STATE UNIV.

\author{
STANFORD UNIVERSITY \\ UNIV. OF BRITISH COLUMBIA \\ UNIV. OF CALIFORNIA, BERKELEY \\ UNIV. OF CALIFORNIA, DAVIS \\ UNIV. OF CALIFORNIA, LOS ANGELES \\ UNIV. OF CALIFORNIA, RIVERSIDE \\ UNIV. OF CALIFORNIA, SAN DIEGO \\ UNIV. OF CALIF., SANTA BARBARA
}

\author{
Daryl Cooper \\ Department of Mathematics \\ University of California \\ Santa Barbara, CA 93106-3080 \\ cooper@math.ucsb.edu \\ Jiang-Hua Lu \\ Department of Mathematics \\ The University of Hong Kong \\ Pokfulam Rd., Hong Kong \\ jhlu@maths.hku.hk \\ Paul Yang \\ Department of Mathematics \\ Princeton University \\ Princeton NJ 08544-1000 \\ yang@math.princeton.edu
}

These supporting institutions contribute to the cost of publication of this Journal, but they are not owners or publishers and have no responsibility for its contents or policies.

See inside back cover or msp.org/pjm for submission instructions.

The subscription price for 2014 is US $\$ 410 /$ year for the electronic version, and \$535/year for print and electronic.

Subscriptions, requests for back issues and changes of subscribers address should be sent to Pacific Journal of Mathematics, P.O. Box 4163, Berkeley, CA 94704-0163, U.S.A. The Pacific Journal of Mathematics is indexed by Mathematical Reviews, Zentralblatt MATH, PASCAL CNRS Index, Referativnyi Zhurnal, Current Mathematical Publications and Web of Knowledge (Science Citation Index).

The Pacific Journal of Mathematics (ISSN 0030-8730) at the University of California, c/o Department of Mathematics, 798 Evans Hall \#3840, Berkeley, CA 94720-3840, is published twelve times a year. Periodical rate postage paid at Berkeley, CA 94704, and additional mailing offices. POSTMASTER: send address changes to Pacific Journal of Mathematics, P.O. Box 4163, Berkeley, CA 94704-0163.

PJM peer review and production are managed by EditFLOW ${ }^{\circledR}$ from Mathematical Sciences Publishers.

\section{PUBLISHED BY}

\section{mathematical sciences publishers \\ nonprofit scientific publishing}

http://msp.org/

(C) 2014 Mathematical Sciences Publishers 


\section{PACIFIC JOURNAL OF MATHEMATICS}

Volume $271 \quad$ No. $1 \quad$ September 2014

Proper holomorphic maps between bounded symmetric domains revisited 1

GAUTAM BHARALI and JAIKRISHNAN JANARDHANAN

An explicit Majorana representation of the group $3^{2}: 2$ of $3 C$-pure type 25

HSIAN-YANG CHEN and CHING HUNG LAM

Sofic groups: graph products and graphs of groups

LAUra Ciobanu, DereK F. Holt and SARAh ReES

Perturbations of a critical fractional equation

Eduardo Colorado, Arturo de Pablo and Urko SÁnchez

A density theorem in parametrized differential Galois theory

THOMAS DREYFUS

On the classification of complete area-stationary and stable surfaces in $\quad 143$ the subriemannian Sol manifold

MATteo Galli

Periodic orbits of Hamiltonian systems linear and hyperbolic at infinity

BAŞAK Z. GÜREL

Nonsplittability of the rational homology cobordism group of

3-manifolds

SE-Goo KIM and CHARLES LIVINGSTON

Biharmonic surfaces of constant mean curvature

ERIC LOUBEAU and CEZAR ONICIUC

Foliations of a smooth metric measure space by hypersurfaces with constant $f$-mean curvature

JUNCHEOL PYO

On the existence of large degree Galois representations for fields of small 243 discriminant

JEREMY ROUSE and FRANK THORNE 